\title{
Le handicap urbain et le déclin de la mortalité en Angleterre et
} au Pays de Galles, 1851-1900

\section{Gerry Kearns}

\section{Citer ce document / Cite this document :}

Kearns Gerry. Le handicap urbain et le déclin de la mortalité en Angleterre et au Pays de Galles, 1851-1900. In: Annales de démographie historique, 1993. pp. 75-105;

doi : 10.3406/adh.1993.1836

http://www.persee.fr/doc/adh_0066-2062_1993_num_1993_1_1836

Document généré le 29/06/2016 


\title{
Résumé
}

L'article est une recherche sur les causes de la mortalité en Angleterre et au pays de Galles dans la deuxième moitié du XIXe siècle, sur leur évolution et sur les différences très marquée entre villes et campagnes : le handicap urbain. Le problème est de bien mesurer les causes de la mortalité, ce qui suppose une recherche plus approfondie que celles qui ont été menées jusqu'ici sur le rôle des différentes maladies et sur la définition des circonscriptions administratives. L'article a donc une caractéristique méthodologique très marquée. Pour mener la recherche, il a fallu en effet construire un échantillon urbain - ce qui est fort difficile, comme le montre l'appendice - et un échantillon rural. La recherche est à continuer : il reste que la différence essentielle pour les adultes entre les villes et les campagnes s'explique par la tuberculose respiratoire. Quant à l'écart de l'espérance de vie, il reste marqué, les campagnes ayant également connu une amélioration de leur mortalité.

\begin{abstract}
This paper is a research on the causes of the mortality in England and Wales during the second half of nineteenth century, their évolution and the very large difference between the cities and the country, what is the urban penalty. The difficulty is the measure of the causes of mortality ; we must achieve a most indepth research on the influence of each disease and on the definition of the administrative districts. Therefore, this paper is caracterized by a great methodological effort. To make the research, it was necessary to construct an urban sample -and it is a particularly difficult work, as it is showed in the appendix-, and a rural sample. The research must be continued, but to-day it is clear that the great difference for the adults between cities and countries must be explained by respiratory tuberculosis. As for the gap in the life expectancy, it is always large, because the country has known also an improvement of his mortality.
\end{abstract}


ANNALES DE DEMOGRAPHIE HISTORIQUE 1993

Société de Démographie Historique - E.H.E.S.S. Paris, 1993

\title{
LE HANDICAP URBAIN ET LE DÉCLIN DE LA MORTALITÉ EN ANGLETERRE ET AU PAYS DE GALLES 1851-1900 ${ }^{1}$
}

\author{
par G. KEARNS
}

\section{Le déclin de la mortalité ${ }^{2}$.}

McKeown et Record ont avancé que le grand recul de la mortalité dans la seconde moitié du XIXe siècle était dû, au moins dans le cas de l'Angleterre et du Pays de Galles, à une amélioration significative de la nourriture, dont la conséquence avait été une chute du nombre des décès dus aux maladies respiratoires et à la tuberculose ${ }^{3}$. Leur analyse soulève en fait trois grands problèmes. Il s'agit en premier lieu de savoir comment a été mesuré le changement survenu dans les taux de mortalité ; en second lieu, de nous demander si, dans ce domaine, la dimension nationale est appropriée pour interpréter les faits ; en troisième lieu, de nous interroger sur la manière de comprendre les changements survenus dans les taux de mortalité de certaines maladies. C'est pourquoi je voudrais présenter dans cet article d'autres méthodes pour mesurer les changements survenus dans la mortalité, définir et décrire les modèles de mortalité dans les zones rurales et urbaines, et, finalement, faire quelques suggestions pour prendre en compte d'une autre manière les changements significatifs que peut présenter l'évolution de la mortalité ${ }^{4}$.

1. Traduction par J.-P. Poussou, qui tient à remercier Madame L. Gallet-Blanchard, professeur à l'Université de Paris-Sorbonne, pour l'aide si précieuse qu'elle lui a apportée pour cette traduction.

2. Je tiens ici à remercier pour leurs conseils mes collègues de Liverpool : Naomi Williams, Graham Mooney, Bob Woods, Paul Laxton et Robert Lee ; mais aussi Julie Holbrooke qui a entré les données dans l'ordinateur, Sandra Mather qui a préparé les illustrations, Ian Qualtrough qui a effectué le travail photographique. Par ailleurs, je dois préciser que le présent article fait partie d'une étude comparative de la mortalité en Suède et en Angleterre, et je tiens à remercier pour leur aide John Rogers et Marie Clarke Nelson de l'Université d'Upsala.

Il a été présenté sous forme de communication à la conférence sur "l'Histoire de la santé publique et de la prévention", qui s'est tenue à Stockholm en septembre 1991 ; je suis redevable à Jan Sundin, qui l'avait organisée, et aux autres participants, pour l'intérêt qu'ils lui ont porté et pour leurs suggestions.

Enfin, je dois souligner que cette recherche a été financée par le Wellcome Trust et que j'ai pu l'écrire grâce à une bourse en Sciences Sociales de la Fondation Nuffield.

3. T. MCKEOWN et R. G. RECORD, "Reason for the decline in mortality", Population Studies, t. XVI, p. 94-122.

4. Le présent article s’intéresse davantage aux différences entrc villcs et campagnes; il s'appuie sur l'analyse des difficultés que l'on rencontre lorsqu'on utilise le tableau national de McKeown, analyse présentée dans G. KEARNS, "The urban penalty and the population history of England", dans A. Brändström et L. G. Tedebrand éd., Society, health and population during the demographic transition, Stockholm, 1988, p. 231-236. 


\section{Mesurer les changements de la mortalité.}

McKeown et Record ont utilisé des taux de mortalité standardisés, tenant compte des spécificités de chaque maladie, pour examiner la structure du déclin de la mortalité au cours de la période 1851-1901. Ils ont construit ces taux standardisés de mortalité à partir des données sur la mortalité contenues dans les suppléments décennaux publiés par le Bureau de l'état civil pour les années 1851-1860 et 1891-1900. Puis ils ont donné le pourcentage de changement des taux d'une mortalité particulière dans le changement d'ensemble survenu à partir de toutes les causes de mortalité, et appelé cela la contribution de chaque maladie à la chute de la mortalité. Or cette approche, comme la plupart de toutes les autres, néglige le problème que, tandis que pour ccrtaines maladies la situation s'améliorait, pour d'autres elle se détériorait, ce qui signifie que l'ampleur de l'amélioration et la part que joue chaque maladie à ce sujet dépendent d'autres travaux concernant l'ensemble ou de changements du taux de mortalité, ce qui ne peut être défini. Par ailleurs, en liant la mortalité à une population moyenne, cette approche ne reconnaît pas que le panorama des maladies change avec l'âge et que des améliorations des causes de la mortalité parmi les jeunes ont un plus grand effet sur l'espérance de vie que la réduction de la mortalité au niveau des personnes âgées. C'est pourquoi je présenterai dans cet article non pas des tableaux du pourcentage de déclin de la mortalité par suite de causes variées, mais les changements absolus survenus dans la durée de vie par suite des changements divers survenus dans la répartition causale de la mortalité par maladies ${ }^{5}$. Néanmoins, pour mieux éclairer le texte, le tableau 1 compare l'importance relative des changements dans les diverses maladies mesurés à partir des modifications survenues dans le taux moyen de mortalité et l'espérance de vie à la naissance.

McKeown et Record ont accordé la plus grande attention à ces "maladies contagieuses qui ont contribué grandement au déclin de la mortalité". Ainsi, par rapport au recul du taux moyen de la mortalité : de 20,48 à $17,630 / 00$, la chute de la mortalité pour la tuberculose respiratoire a été de 2,76 à 1,41 , ce qui représente $43,3 \%$ de la chute globale. Et si l'on ajoute à cela la prise en compte de l'ensemble de la chute concernant la tuberculose, on en arrive à $47 \%$, ce qui justifie l'assertion de McKeown et Brown que près de la moitié de la chute de la mortalité a été due à ce qu'ils identifient comme des maladies dont l'évolution est directement commandée par celle de la nourriture. Or, lorsqu'on prend en compte la distribution par âges de la mortalité, le rôle de la tuberculose respiratoire apparaît plutôt moins grand : la chute de la mortalité due à cette cause particulière n'ajoute plus que 1,81 année à l'espérance de vie à la naissance, soit $35,3 \%$ de l'amélioration de cette espérance de vie. L'autre cause de mortalité dont l'importance recule également est le groupe de diarrhées.

\section{Les différences entre villes et campagnes et le changement de la mortalité.}

R. Woods a montré à quel point notre interprétation de l'échelle du recul de la mortalité peut être affectée par l'unité d'analyse choisie ${ }^{6}$. Puisqu'il y eut, au cours

5. Cette approche est celle qui a été déjà choisie dans S. H. Preston, N. Keyfitz et R. SCHOEN, Causes of death: life tables for national populations, New York, 1972. J'ai à exprimer de très grands remerciements à Graham Mooney pour l'aide qu'il m'a apportée dans le calcul des tables de mortalité.

6. R. Woods, "The effect of population redistribution on the level of mortality in nineteenth century England and Wales", Journal of Economic History, t. XLV, 1985, p. 645-651. 
Tableau 1. - Taux de mortalité standardisés (décès pour 1000 naissances vivantes) et rôle des différentes causes de décès dans les changements survenus dans l'espérance de vie : 1851-1860 et 1891-1900.

Cause de décès

1851-1860 1891-1900 changement

Changement

$\% \mathrm{du}$

en espérance

ngement total de vie total

A. Maladies contagieuses contribuant fortement au déclin de la mortalité

\begin{tabular}{|c|c|c|c|c|}
\hline Tuberculose respiratoire & 2.76 & 1.41 & 43.3 & 1.81 \\
\hline Autres tuberculoses & 0.69 & 0.57 & 3.7 & 0.20 \\
\hline Typhys et autres fièvres & 0.89 & 0.18 & 22.7 & 0.97 \\
\hline Scarlatine & 0.78 & 0.15 & 20.0 & 1.07 \\
\hline Diarrhées & 0.92 & 0.63 & 9.4 & 0.26 \\
\hline Total A. & 6.03 & 2.94 & 99.1 & 4.52 \\
\hline
\end{tabular}

B. Quelques autres maladies contagieuses

$\begin{array}{lccccr}\text { Variole } & 0.20 & 0.01 & 5.8 & 0.30 & 5.9 \\ \text { Coqueluche } & 0.41 & 0.34 & 2.3 & 0.12 & 2.4 \\ \text { Rougeole } & 3.49 & 3.85 & -1.2 & -0.07 & -1.3 \\ \text { Diphtérie } & 0.10 & 0.25 & -4.9 & -0.26 & -5.1 \\ \text { Total B. } & 10.5 & 0.99 & 2.0 & 0.10 & 1.9\end{array}$

C. Autres causes de maladies retenues par Mc Keown et Record

$\begin{array}{lllcrr}\text { Maladies cérébrales } & 2.41 & 2.05 & 11.4 & 0.78 & 15.2 \\ \text { Maladies cardiaques } & 1.27 & 1.67 & -12.6 & -0.25 & -4.9 \\ \text { Maladies pulmonaires } & 2.77 & 3.25 & -15.5 & -0.54 & -10.5 \\ \text { Maladies stomacales } & 0.99 & 1.12 & -4.0 & -0.43 & -8.4 \\ \text { Maladies rénales } & 0.22 & 0.46 & -7.8 & -0.22 & -4.4 \\ \text { Total C } & 7.66 & 8.55 & -28.5 & -0.64 & -12.5\end{array}$

D. Autres causes:

Cancers

0.33

0.75

("zymotics")

Maladies héréditaires

Maladies articulaires

0.06

0.07

Maladies de la peau

0.04

Morts lors des accouchements

Morts violentes

Total D.

0.17

0.16

0.65

4.89

5.74

20.48
$-14.0$

$-0.35$

$-6.9$

(21.3)

(1.09)

0.01

0.2

(0.09)

(1.7)

(1.4)

(0.06)

0.02

0.4

0.11

1.20

23.6 
de XIXe siècle, un accroissement considérable de la part de la population vivant dans les zones urbaines où la mortalité était en données relatives plus forte qu'ailleurs, le taux moyen de mortalité a dâ s'améliorer aussi bien en ville qu'à la campagne pour que la moyenne nationale reste simplement constante.Ce handicap urbain rend évident qu'effectivement il a fallu une nette amélioration à la campagne pour que le taux national reste constant. De ce fait, la moyenne nationale peut n'être qu'un guide inapproprié pour mesurer l'échelle et le changement de la mortalité, ce qui est une conclusion particulièrement importante pour notre interprétation des causes de la diminution de la mortalité.

Les caractéristiques générales du handicap urbain ont été décrites ailleurs ${ }^{7}$; elles incluent une courbe en " $U$ ", exagérée, de la mortalité, les jeunes et les vieux étant spécialement placés en ville dans les conditions les plus pauvres, et une surmortalité très forte des hommes par rapport aux femmes des mêmes âges. À partir des informations sur l'espérance de vie tirées des registres d'état civil de 600 districts pour la période 1861-1863, nous pouvons nous faire une idée générale des différences significatives qui existent sur les graphiques entre les villes et les campagnes $^{8}$. La figure I montre que les niveaux de la mortalité infantile sont plus

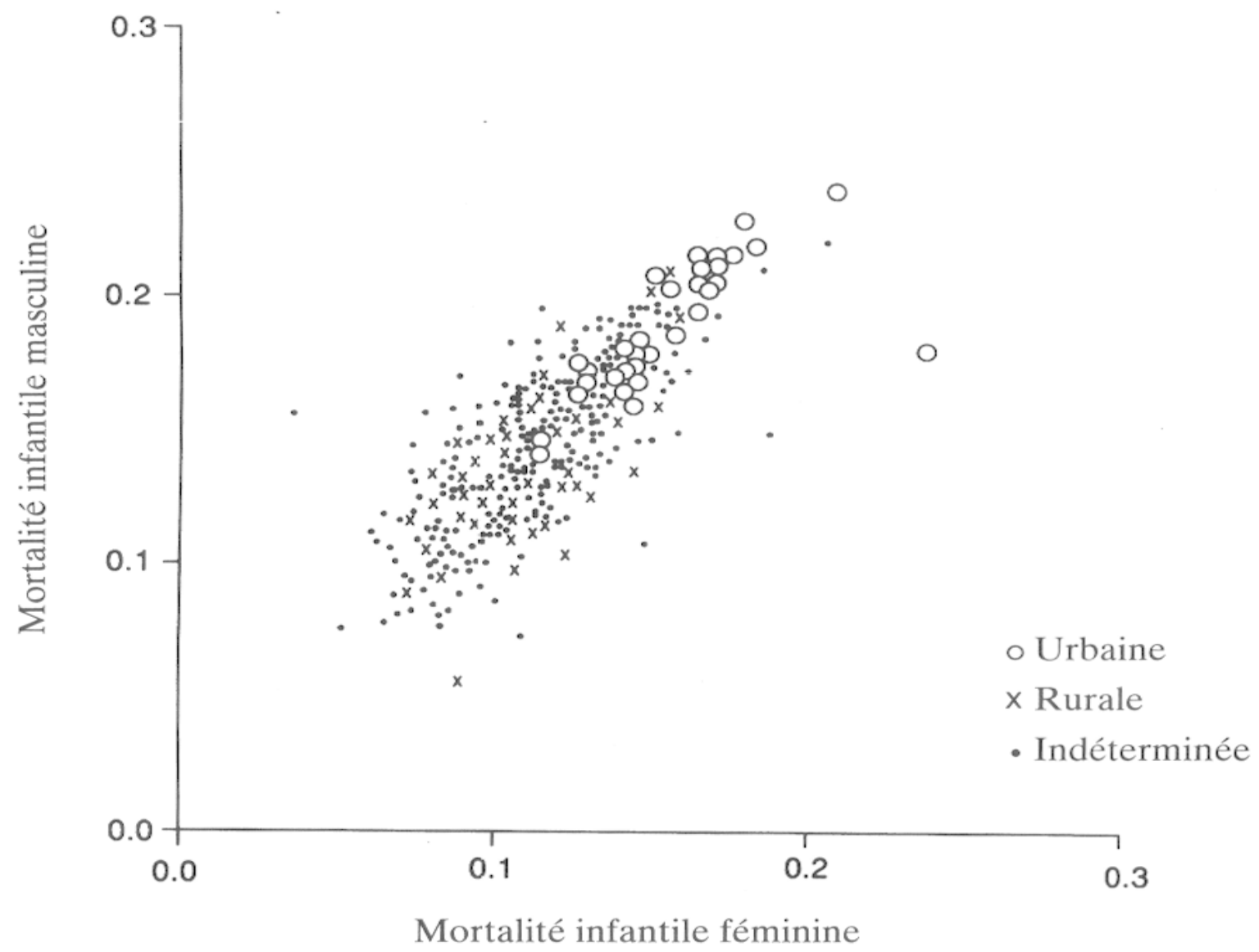

Figure I. - Mortalité infantile masculine et féminine 1861-1863:

Districts urbains, ruraux et autres districts d'enregistrement

7. Voir G. KEARNS, "Biology, class and the urban penalty", dans G. Kearns et C. J. Withers éd., Urbanising Britain: essays on class and Community in the nineteenth century, Cambridge, 1991, p. 12-30. 
élevés dans les zones urbaines, ainsi que l'excédent, normal du point de vue biologique, des taux masculins sur les taux féminins. La figure II indique que l'espérance de vie à 20 ans était plus haute à la campagne qu'à la ville. A la différence des villes, à la campagne les hommes qui avaient atteint l'âge de 20 ans vivaient plus longtemps que les femmes. Quels que soient les éléments contrôlant l'espérance de vie à 20 ans dans les milieux ruraux, la figure III révèle qu'il y avait peu de corrélations entre ces facteurs et ceux qui commandaient la mortalité infantile dans les mêmes zones. Il n'y avait donc pas de corrélation entre la mortalité infantile et l'espérance de vie à la naissance dans les districts ruraux, alors qu'au contraire la figure IV montre qu'elle était très étroite en ville. Les facteurs qui expliquent la mortalité infantile supérieure des villes par rapport à celles des campagnes sont étroitement liés à ceux qui expliquent les chiffres supérieurs de la mortalité urbaine des adultes, encore que la différence concerne surtout les hommes.

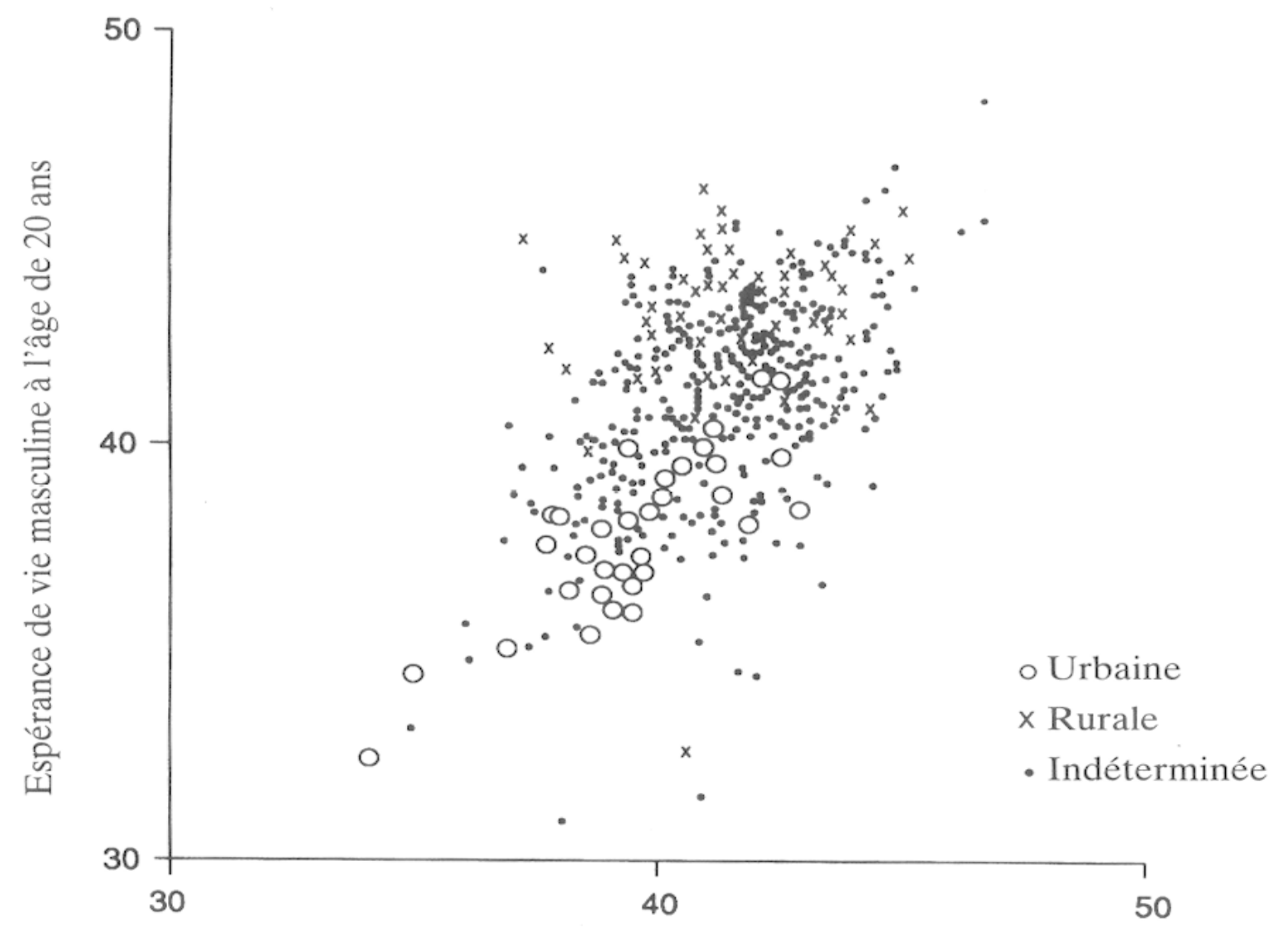

Espérance de vie féminine à l'âge de 20 ans

Figure II. - Espérance de vie à l'âge de 20 ans pour les hommes et les femmes en 1861-1863

8. Je remercie Bob Woods pour ces données qui sont décrites et analysées dans R. WooDs, "The structure of mortality in mid-nineteenth century England and Wales", Journal of Historical Geography, t. VIII, 1982, p. 373-394. Pour prendre en compte les districts d'enregistrement urbains, ruraux, et ceux correspondant aux groupements intermédiaires, $j$ 'ai appliqué à ces données la classification d'enregistrement présentée ci-après. 


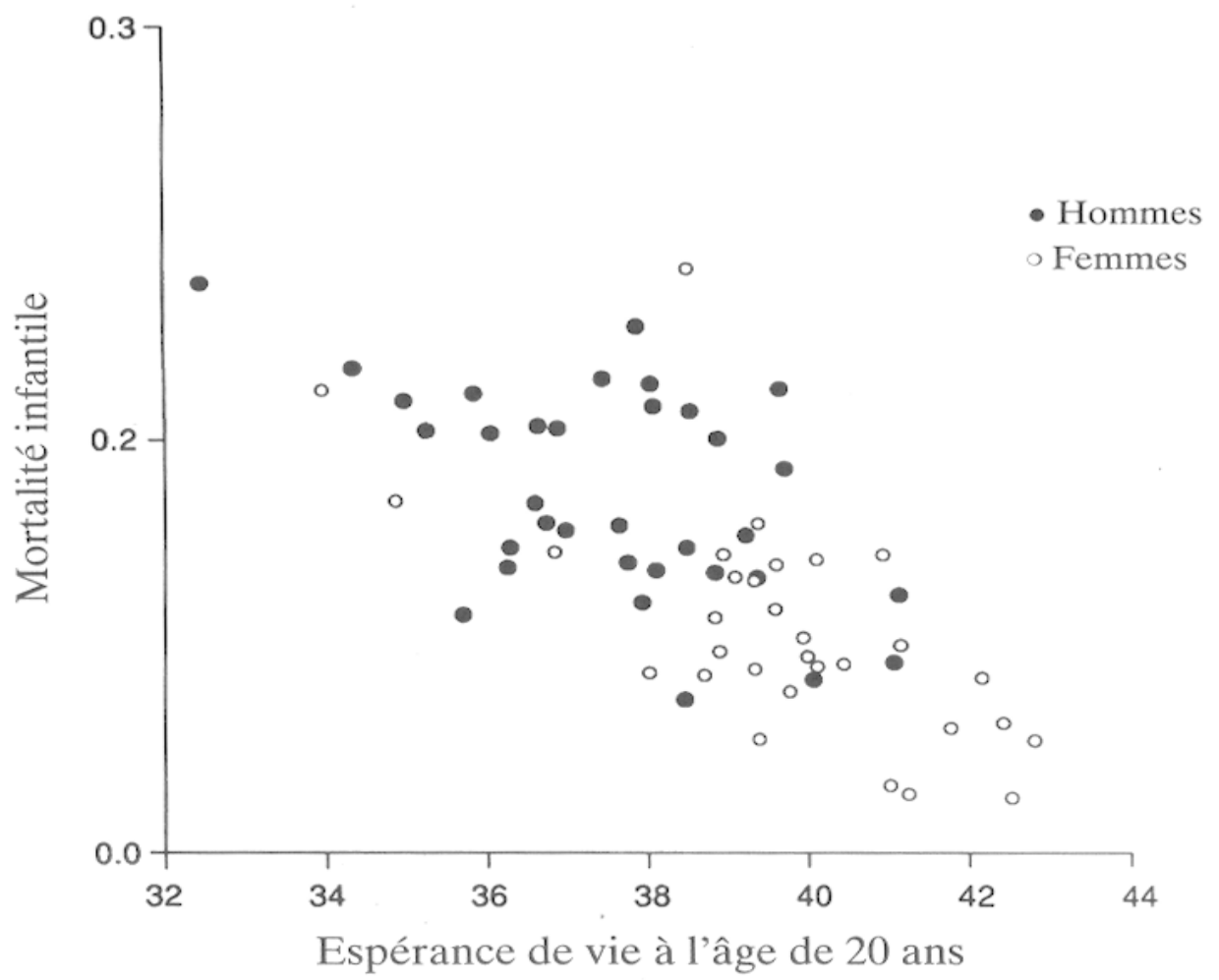

Figure III. - Mortalité infantile et espérance de vie à l'âge de 20 ans. Hommes et femmes : districts ruraux 1861-1863.

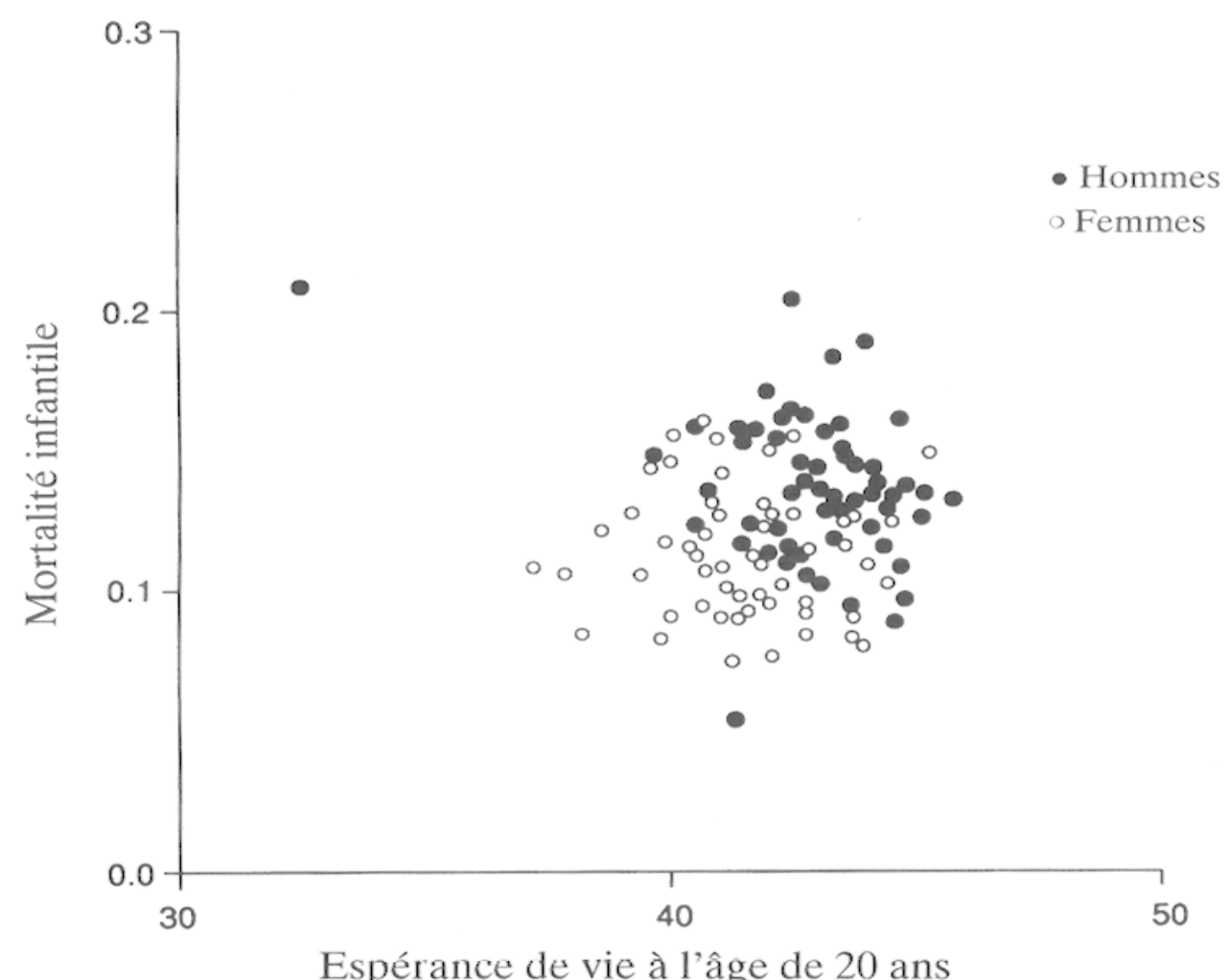

Figure IV. - Mortalité infantile et espérance de vie à l'âge de 20 ans. Hommes et femmes : districts urbains d'enregistrement 1861-1863. 
Pour étudier plus en profondeur ces contrastes, nous devons analyser l'interaction qui existe entre la mortalité spécifique par âges et celle suivant les maladies puisque nous allons devoir expliquer les raisons qui expliquent les différences dans ce recul et les changements qui concernent leur extension. L'analyse de McKeown n'a pas prêté suffisamment attention à tous les facteurs différentiels liés à l'âge ou au contraste villes-campagnes pour décrire ou expliquer le grand recul de la mortalité.

\section{Les districts urbains et ruraux.}

\section{Définir l'échantillon urbain.}

Les matériaux publiés par le Bureau de l'état civil sont une source tout à fait valable pour étudier la démographie des villes anglaises dans la deuxième moitié du XIXe siècle. Pour l'essentiel, ils proviennent des données d'état civil concernant 600 districts d'Angleterre et du pays de Galles ${ }^{9}$. Cela impose de sérieuses limites quant à la sophistication avec laquelle des zones urbaines sont délimitées par ceux qui étudient la démographie des villes. Il est possible, à partir des volumes publiés à partir des recensements successifs, de connaître les changements totaux de population de tout un ensemble d'unités administratives aussi bien modernes qu'anciennes : "boroughs", "township", "hundreds", paroisses et ainsi de suite ${ }^{10}$. Utilisés avec les données qu'apportent les cartes retraçant le développement des zones bâties de chaque ville ou cité, ces matériaux permettent de mesurer de manière détaillée la population des unités urbaines quelle que soit celle que l'on a choisie comme objet d'étude. C'est ainsi que Law a établi ses estimations de la population anglaise vivant dans des villes et cités de tailles différentes au cours du XIXe siècle ${ }^{11}$. Parce que nous devons travailler à partir des districts d'enregistrement, nous ne pouvons pas utiliser dans le détail les richesses des recensements publiés. Il nous faut essentiellement nous poser une question : quel groupe de districts d'enregistrement complet permet de définir le mieux possible chaque ville en particulier?

L'échantillon choisi ici est celui des villes ayant en 1851 une population de 50000 habitants ou plus. Londres devait être placée comme il convenait dans une

9. Quand le Bureau du Conservateur général des actes de l'état civil ou Bureau de l'état civil ("General Register office", souvent écrit en abrégé G. R. O. - N. d. T.) fut créé en 1838, les syndicats de la "Loi des Pauvres" ("Poor Law Unions") créés en 1834 par "La loi révisant la loi sur les Pauvres" ("Poor Law Amendment Act") furent utilisés comme divisions de base pour un système formé par 324 districts d'enregistrement.Ceux-ci furent très vite portés à 623 et ils continuèrent à former la base du système d'enregistrement pour le reste du siècle, jusqu'à ce qu'un nouveau regroupement des districts de santé ruraux et urbains soit adopté pour être en harmonie avec un nouveau système de gouvernement local.

10. Les "boroughs" sont des villes dotées d'une municipalité ; les "townships" des communes qui sont le plus souvent des petites villes ou bourgs ; les "hundreds" des parties de communes. II est toujours préférable d'employer le terme anglais car nous n'avons pas exactement le mot français correspondant. Il est question de "county borough" ou "ville-comté" ; c'est le statut le plus élevé pour une ville car elle est chef-lieu de comté. Quant au terme "municipal borough", il désigne une grande ville qui, n'ayant pas le statut de ville-comté, dépend encore de l'autorité du conseil de comté. En effet, il y a opposition fondamentale, dans l'administration locale, entre les comtés ("counties") et les villes ou bourgs-comtés ("county-boroughs").

Voir à ce sujet M. CHARLOT éd., Encyclopédie de la Civilisation britannique, Paris, Larousse, 1976 ; et, notamment pour son lexique, C. MoINDroT, Villes et Campagnes britanniques, Paris, A. Colin, Coll. U2, 1967. (N. d. T.).

11. C. M. LAw, "The growth of the urban population of England and Wales 1801-1911", Transactions of the Institute of British Geographers, t. XLI, 1967, p. 125-143. 
catégorie particulière et, pour elle, l'unité la plus adéquate était probablement le district d'enregistrement ; l'article n'étudie donc que les grandes villes en dehors de Londres" 12 . Pour ces villes, le "municipal borough" doit être considéré comme l'unité délimitant la taille de la ville réelle. Nous avons donc pu faire entrer dans notre échantillon urbain ${ }^{13} 22$ villes : Bath, Birmingham, Bolton, Bradford, Brighton, Bristol, Hull, Leeds, Leicester, Liverpool, Manchester, Newcastle-uponTyne, Norwich, Nottingham, Oldham, Plymouth, Portsmouth, Preston, Sheffield, Stockport, Stoke-on-Trent et Sunderland ${ }^{14}$. En utilisant les notes qui figurent dans les volumes publiés des recensements de 1851 et de 1901 , ainsi que les autres publications qui les concernent, nous allons essayer de bâtir notre échantillon urbain, considérant individuellement chaque ville.

En construisant notre échantillon urbain, nous n'avons pas voulu créer une fausse opposition entre la ville et la campagne, ce qui aurait pu arriver en incluant seulement les centres urbains des grandes conurbations. Au cours du temps cela serait devenu de manière croissante le cas si nous avions seulement sélectionné les districts d'enregistrement portant le même nom que les villes auxquelles ils correspondent. D'une manière similaire, nous n'avons pas voulu diluer notre échantillon urbain dans des zones rurales très étendues, simplement dans le but de ne pas laisser échapper les populations relativement petites de la plupart des zones périphériques de banlieue ${ }^{15}$. Mais, approximativement, nous n'avons inclus aucun district d'enregistrement dont moins de la moitié de la population se trouvait à l'intérieur d'une ville.

Pour l'année 1851, les résultats figurent dans le tableau $2^{16}$. Globalement, ils sont largement satisfaisants. Les districts d'enregistrement choisis montrent une très étroite corrélation avec la population des "municipal boroughs" 17 . Seules

12. Graham Mooney, qui appartient au Département de Géographie de l'Université de Liverpool, fait des recherches sur la démographie de Londres au XIXe siècle : nous travaillons ensemble à un article qui comparera Londres et les villes provinciales.

13. Cela n'a pu être réalisé sans problèmes puisque des villes qui furent créées à travers la coalescence de l'expansion urbaine ont pu passer à travers le crible des villes parlementaires ou de celles dotées de municipalités. La seule manière pour faire confiance à notre sélection a été de choisir un niveau de découpage très élevé, et donc tel qu'il ne puisse y avoir que peu de candidates et qu'elles soient bien définies, et d'avoir recours à la cartographie pour tous les cas douteux. En fait, c'est une tâche qui est loin d'être facile tant que nous ne disposons pas d'un atlas convenable des zones urbaines de développement au XIXe siècle. Les régions urbaines du sudouest du Lancashire, du West Riding du Yorkshire et le district des Poteries sont celles qui présentent le plus de difficultés ; c'est là que les choix opérés pourront être le plus sujets à révision.

14. Cette sélection de villes a été discutée plus en détail dans G. KEARNS, Aspects of cholera, society and space in nineteenth-century England and Wales, thèse non publiée de doctorat (Ph. D.), Université de Cambridge, 1985, chap. II ; et dans N. WILliaMs, Infant and child mortality in urban areas of nineteenth-century England and Wales : a record-linkage study, thèse de doctorat non publiée, Université de Sheffield, 1989, Appendice 1.

L'actuelle sélection des districts d'enregistrements diffère quelque peu de ces premiers travaux. La ville "parlementaire" (L'expression de "parliamentary borough" désigne des villes qui envoient un député au Parlement - N. d. T.) de Merthyr Tydfil a une population de 63080 habitants mais la carte suggère qu'elle comprend toujours en 1851 une importante proportion de population rurale ; en outre, le district d'enregistrement de Merthyr Tydil fut sérieusement surdélimité à cette date, et j'ai donc été forcé, à mon grand regret, d'exclure cette cité galloise de mes analyses. - Je suis très reconnaissant envers Paul Laxton et Naomi Williams pour l'aide qu'ils m'ont apportée dans ce domaine.

15. J'ai traduit par "banlieue", ce qui convient bien lorsque le mot "suburbs" est pris comme ici dans un sens général ; il n'en reste pas moins qu'à bien des égards les "suburbs" anglais sont fort différents des banlieues françaises (N. d. T.).

16. Les villes "réelles" considérées ici sont toutes des villes dotées de municipalités ("Municipal boroughs", N. d. T.), sauf lorsqu'il n'y avait pas de municipalité en exercice ; dans ce cas les villes envoyant un député au parlement ("parliamentary borough" (N. d. T.) ont été choisies, comme cela a été indiqué sur le tableau.

17. "Municipal borough" dans le texte. - Voir notes 10 et 16 (N. d. T.). 
Tableau 2. - Définition des villes retenues en 1851.

\begin{tabular}{|c|c|c|c|c|c|c|c|c|c|}
\hline Ville ("borough") & Population & $\begin{array}{c}\text { Population } \\
\text { n'appartenant } \\
\text { pas aux districts } \\
\text { d'enregistrement }\end{array}$ & $\begin{array}{l}\% \text { de la } \\
\text { population } \\
\text { dans ce cas }\end{array}$ & $\begin{array}{c}\text { Districts } \\
\text { d'enregistrement }\end{array}$ & Population & $\begin{array}{l}\text { Population } \\
\text { non située } \\
\text { dans la ville }\end{array}$ & $\begin{array}{c}\% \text { de la } \\
\text { population se } \\
\text { trouvant dans } \\
\text { ce cas }\end{array}$ & $\begin{array}{l}\text { Population } \\
\text { apparemment } \\
\text { attachée à } \\
\text { la ville }\end{array}$ & $\begin{array}{c}\% \text { de la } \\
\text { population se } \\
\text { trouvant dans } \\
\text { ce cas }\end{array}$ \\
\hline Bath & 54240 & 0 & 0.00 & 326.Bath & 69847 & 15607 & 22.34 & 8000 & 11.45 \\
\hline \multirow[t]{3}{*}{ Birmingham } & 232841 & 0 & 0.00 & 393.Kings Norton & 30871 & 21602 & 69.98 & 8379 & 27.14 \\
\hline & & & & 394.Birmingham & 173951 & 0 & 0.00 & & \\
\hline & & & & 395.Aston & 66852 & 17231 & 25.77 & 11660 & 17.44 \\
\hline Bolton & 61171 & 0 & 0.00 & 468.Bolton & 114712 & 53541 & 46.67 & 0 & 0.00 \\
\hline Bradford & 103778 & 0 & 0.00 & 499.Bradford & 181964 & 78186 & 42.97 & 0 & 0.00 \\
\hline Brighton (P) & 69673 & 4104 & 5.89 & 85.Brighton & 65569 & 0 & 0.00 & & \\
\hline \multirow[t]{2}{*}{ Bristol } & 137328 & 17598 & 12.81 & 329.Bristol & 65716 & 0 & 0.00 & & \\
\hline & & & & 330.Clifton & 77950 & 23936 & 30.71 & 1221 & 1.57 \\
\hline \multirow[t]{2}{*}{ Hull } & 84690 & 0 & 0.00 & 519.Sculcoates & 44719 & 9026 & 20.18 & 0 & 0.00 \\
\hline & & & & 520.Hull & 50670 & 0 & 0.00 & & \\
\hline \multirow[t]{2}{*}{ Leeds } & 172270 & 0 & 0.00 & 500.Hunslet & 88679 & 17752 & 20.02 & 0 & 0.00 \\
\hline & & & & 501.Leeds & 101343 & 0 & 0.00 & & \\
\hline Leicester & 60584 & 0 & 0.00 & 417.Leicester & 60642 & 58 & 0.10 & 0 & 0.00 \\
\hline \multirow[t]{3}{*}{ Liverpool } & 375955 & 0 & 0.00 & 460.Wirral & 57157 & 57157 & 100.00 & 39143 & 68.48 \\
\hline & & & & 461.Liverpool & 258236 & 0 & 0.00 & & \\
\hline & & & & 462. West Derby & 153279 & 34167 & 22.29 & 13621 & 8.89 \\
\hline Manchester & 303382 & 0 & 0.00 & 471.Chorlton & 123841 & 19024 & 15.36 & 0 & 0.00 \\
\hline and & & & & 473.Manchester & 228433 & 29868 & 13.08 & 0 & 0.00 \\
\hline Salford & 63850 & 0 & 0.00 & 472.Salford & 87523 & 23673 & 27.05 & 0 & 0.00 \\
\hline Merthyr Tydfil (P) & 63080 & 0 & 0.00 & 582.Merthyr Tydfil & 76804 & 13724 & 17.87 & 0 & 0.00 \\
\hline Newcastle-upon-Tyne & 87784 & 0 & 0.00 & 552.Newcastle-upon-Tyne & 89156 & 1372 & 1.54 & 0 & 0.00 \\
\hline and Gateshead & 25568 & 0 & 0.00 & 551.Gateshead & 48081 & 22513 & 46.82 & 0 & 0.00 \\
\hline Norwich & 68195 & 0 & 0.00 & 234.Norwich & 68195 & 0 & 0.00 & & \\
\hline Nottingham & 57407 & 0 & 0.00 & 440.Nottingham & 58419 & 1012 & 1.73 & 1012 & 1.73 \\
\hline Oldham & 52820 & 0 & 0.00 & 475.Oldham & 86788 & 33968 & 39.14 & 0 & 0.00 \\
\hline Plymouth & 52221 & 0 & 0.00 & 287.Plymouth & 52221 & 0 & 0.00 & & \\
\hline Portsmouth & 72096 & 0 & 0.00 & 96.Portsea Island & 72126 & 30 & 0.04 & 30 & 0.04 \\
\hline Preston & 69542 & 0 & 0.00 & 482.Preston & 96545 & 27003 & 27.97 & 0 & 0.00 \\
\hline \multirow[t]{2}{*}{ Sheffield } & 135510 & 0 & 0.00 & 507.Eccleshall Bierlow & 37914 & 2966 & 7.82 & 0 & 0.00 \\
\hline & & & & 508.Sheffield & 103626 & 3264 & 3.15 & 0 & 0.00 \\
\hline Stockport & 53835 & 0 & 0.00 & 452.Stockport & 90208 & 36373 & 40.32 & 0 & 0.00 \\
\hline Stoke-upon-Trent (P) & 84027 & 28675 & 34.13 & 371.Stoke-upon-Trent & 57942 & 8102 & 13.98 & 0 & 0.00 \\
\hline Sunderland & 63897 & 0 & 0.00 & 549.Sunderland & 70576 & 6679 & 9.46 & 0 & 0.00 \\
\hline Les grandes villes & 2605744 & 50377 & 1.93 & & 3110555 & 557834 & 17.93 & 83066 & 2.67 \\
\hline
\end{tabular}


Tableau 3. - Définitions des villes retenues en 1891.

\begin{tabular}{|c|c|c|c|c|c|c|c|c|c|}
\hline Ville ("borough") & Population & $\begin{array}{c}\text { Population } \\
\text { n'appartenant } \\
\text { pas aux districts } \\
\text { d'enregistrement }\end{array}$ & $\begin{array}{c}\% \text { de la } \\
\text { population } \\
\text { dans ce cas }\end{array}$ & $\begin{array}{c}\text { Districts } \\
\text { d'enregistrement }\end{array}$ & Population & $\begin{array}{l}\text { Population } \\
\text { non située } \\
\text { dans la ville }\end{array}$ & $\begin{array}{c}\% \text { de la } \\
\text { population se } \\
\text { trouvant dans } \\
\text { ce cas }\end{array}$ & $\begin{array}{l}\text { Population } \\
\text { apparemment } \\
\text { attachée à } \\
\text { la ville }\end{array}$ & $\begin{array}{c}\% \text { de la } \\
\text { population se } \\
\text { trouvant dans } \\
\text { ce cas }\end{array}$ \\
\hline Bath & 49839 & 0 & 0.00 & 317.Bath & 77604 & 27765 & 35.78 & 17061 & 21.98 \\
\hline \multirow[t]{4}{*}{ Birmingham } & 522204 & 0 & 0.00 & 385.King's Norton & 187087 & 111661 & 59.68 & 54539 & 29.15 \\
\hline & & & & 386.Birmingham & 245216 & 0 & 0.00 & & \\
\hline & & & & 387.Aston & 312263 & 110701 & 35.45 & 16368 & 5.24 \\
\hline & & & & 394.Solihull & 48618 & 48618 & 100.00 & 33946 & 69.82 \\
\hline Bolton & 168215 & 0 & 0.00 & 461:Bolton & 257587 & 89372 & 34.70 & 25925 & 10.06 \\
\hline \multirow[t]{2}{*}{ Bradford } & 279767 & 0 & 0.00 & 497a.Bradford & 228625 & 0 & 0.00 & & \\
\hline & & & & 497b.North Bierley & 129067 & 77925 & 60.38 & 25573 & 19.81 \\
\hline \multirow[t]{2}{*}{ Brighton } & 123478 & 0 & 0.00 & 77.Brighton & 102320 & 0 & 0.00 & & \\
\hline & & & & 78.Steyning & 80789 & 59631 & 73.81 & 29695 & 36.76 \\
\hline \multirow[t]{3}{*}{ Bristol } & 328945 & 0 & 0.00 & 319.Long Ashton & 24138 & 24138 & 100.00 & 0 & 0.00 \\
\hline & & & & 320.Bristol & 328945 & 0 & 0.00 & & \\
\hline & & & & 321.Barton Regis & 16502 & 16502 & 100.00 & 0 & 0.00 \\
\hline \multirow[t]{2}{*}{ Hull } & 240259 & 0 & 0.00 & 520.Sculcoates & 173307 & 15293 & 8.82 & 0 & 0.00 \\
\hline & & & & 521.Hull & 82245 & 0 & 0.00 & & \\
\hline \multirow[t]{4}{*}{ Leeds } & 428968 & 0 & 0.00 & 498.Hunslet & 83002 & 13559 & 16.34 & 0 & 0.00 \\
\hline & & & & 499.Holbeck & 33585 & 2013 & 5.99 & 0 & 0.00 \\
\hline & & & & 500.Bramley & 79700 & 3343 & 4.19 & 0 & 0.00 \\
\hline & & & & 501.Leeds & 254536 & 3210 & 1.26 & 1842 & 0.72 \\
\hline Leicester & 211579 & 0 & 0.00 & 409.Leicester & 211579 & 0 & 0.00 & & \\
\hline \multirow[t]{3}{*}{ Liverpool } & 684958 & 0 & 0.00 & 453.Liverpool & 147405 & 0 & 0.00 & & \\
\hline & & & & 454.Toxteth Park & 136230 & 0 & 0.00 & & \\
\hline & & & & 455.West Derby & 529684 & 69805 & 13.18 & 69805 & 13.18 \\
\hline
\end{tabular}




\begin{tabular}{|c|c|c|c|c|c|c|c|c|c|}
\hline Ville ("borough") & Population & $\begin{array}{c}\text { Population } \\
\text { n'appartenant } \\
\text { pas aux districts } \\
\text { d'enregistrement }\end{array}$ & $\begin{array}{l}\% \text { de la } \\
\text { population } \\
\text { dans ce cas }\end{array}$ & $\begin{array}{c}\text { Districts } \\
\text { d'enregistrement }\end{array}$ & Population & $\begin{array}{c}\text { Population } \\
\text { non située } \\
\text { dans la ville }\end{array}$ & $\begin{array}{c}\% \text { de la } \\
\text { population se } \\
\text { trouvant dans } \\
\text { ce cas }\end{array}$ & $\begin{array}{l}\text { Population } \\
\text { apparemment } \\
\text { attachée à } \\
\text { la ville }\end{array}$ & $\begin{array}{c}\% \text { de la } \\
\text { population se } \\
\text { trouvant dans } \\
\text { ce cas }\end{array}$ \\
\hline$\overline{\text { Birkenhead }}$ & 110915 & 0 & 0.00 & 452.Birkenhead & 165171 & 54256 & 32.85 & 4169 & 2.52 \\
\hline \multirow[t]{3}{*}{ Manchester } & 543872 & 0 & 0.00 & 464.Chorlton & 342643 & 0 & 0.00 & & \\
\hline & & & & 466.Manchester & 132136 & 0 & 0.00 & & \\
\hline & & & & 467.Prestwich & 196832 & 12839 & 6.52 & 12839 & 6.52 \\
\hline Salford & 220957 & 0 & 0.00 & 465.Salford & 229450 & 8493 & 3.70 & 0 & 0.00 \\
\hline Merthyr Ty dfil & 69228 & 0 & 0.00 & 588.Merthyr Tydfil & 135540 & 66312 & 48.92 & 0 & 0.00 \\
\hline Newcastle upon Tyne & 215328 & 0 & 0.00 & 556.Newcastle-upon-Tyne & 233644 & 18316 & 7.84 & 0 & 0.00 \\
\hline Gateshead & 109888 & 0 & 0.00 & 555.Gateshead & 166844 & 56956 & 34.14 & 0 & 0.00 \\
\hline Norwich & 111733 & 0 & 0.00 & 225.Norwich & 111733 & 0 & 0.00 & & \\
\hline Nottingham & 239743 & 0 & 0.00 & 431.Nottingham & 239743 & 0 & 0.00 & & \\
\hline Oldham & 137246 & 0 & 0.00 & 469.Oldham & 215624 & 78378 & 36.35 & 24892 & 11.54 \\
\hline \multirow[t]{2}{*}{ Plymouth } & 107636 & 0 & 0.00 & 277.Plymouth & 107636 & 0 & 0.00 & & \\
\hline & & & & 278.East Stonehouse & 15111 & 0 & 0.00 & 15111 & 100.00 \\
\hline Devonport & 70437 & 0 & 0.00 & 279.Devonport & 70437 & 0 & 0.00 & & \\
\hline \multirow[t]{2}{*}{ Portsmouth } & 188133 & 0 & 0.00 & 88.Portsmouth & 188133 & 0 & 0.00 & & \\
\hline & & & & 89.Alverstoke & 28884 & 28884 & 100.00 & 28884 & 100.00 \\
\hline Preston & 112989 & 0 & 0.00 & 476.Preston & 152231 & 39242 & 25.78 & 0 & 0.00 \\
\hline \multirow[t]{2}{*}{ Sheffield } & 380793 & 0 & 0.00 & 508.Ecclesall Bierlow & 179676 & 14176 & 7.89 & 10828 & 6.03 \\
\hline & & & & 509.Sheffield & 229454 & 14161 & 6.17 & 757 & 0.33 \\
\hline Stockport & 78897 & 0 & 0.00 & 443.Stockport & 156308 & 77411 & 49.52 & 16622 & 10.63 \\
\hline Stoke upon Trent & 30458 & 0 & 0.00 & 363.Stoke-upon-Trent & 155422 & 124964 & 80.40 & 84341 & 54.27 \\
\hline Sunderland & 146077 & 0 & 0.00 & 553.Sunderland & 181506 & 35429 & 19.52 & 0 & 0.00 \\
\hline Les grandes villes & 5912542 & 0 & 0.00 & & 7404192 & 1303353 & 17.60 & 473197 & 6.39 \\
\hline
\end{tabular}


Brighton, Bristol et Stoke laissent échapper une partie de leur population hors de leurs districts. Plusieurs districts d'enregistrement couvrent une superficie bien plus large que les "municipal boroughs" 17 qui nous intéressent, mais il ne s'agit que de quelques cas qui correspondent aux zones urbaines qui font partie d'une aire urbaine continue et non de la définition administrative d'une ville ${ }^{17}$. Le seul véritable problème est Merthyr Tydfil qui a été exclue à regret mais nécessairement. Un travail cartographique complémentaire nous permettra d'accroître les chiffres des populations situées en dehors des agglomérations ${ }^{18}$ que nous pourrions considérer comme faisant partie des ces villes ; pour le moment, nous pouvons estimer que la population résiduelle se trouvant en dehors des villes mais cnglobée dans les districts d'enregistrement représente un sixième de la population totale.

En 1901, la situation est un peu plus difficile : l'extension des conurbations peut être appréciée seulement à travers l'examen des évidences cartographiques car les définitions qu'apportent les unités administratives sont moins fiables. L'apport de ces cartes a été augmenté par les données et discussions contenues dans le rapport sur le gouvernement local établi en 1888 par les "Commissaires responsables des découpages administratifs et électoraux" 19 . On en trouve les résultats dans le tableau 3 qui montre que les "City boroughs" 20 ont en 1901 une population de 5912542 habitants cependant que celle des districts d'enregistrement considérés est de 7404192 de personnes, parmi lesquelles 473197 peuvent être considérées comme relevant des villes bien que se trouvant en dehors des "municipal boroughs" 20

\section{Définir "l'échantillon rural".}

Nous avons considéré comme ruraux tous les districts d'enregistrement dont plus de $50 \%$ de la population rurale masculine était employée dans l'agriculture dans le recensement de $1851^{21}$. Il nous a semblé que c'était une définition qui convenait mieux que, par exemple, celle basée sur les densités, laquelle serait trop affectée par la dispersion de population de grandes zones urbaines, certes concentrées mais aussi englobées dans des zones rurales très étendues et faiblement peuplées. En fait, il n'y a aucune méthode permettant de définir de manière réellement adéquate la population urbaine totale de chaque district, bien qu'un travail intensif sur la distribution de la population des villes plus petites aurait pu nous amener en quelque façon vers une définition similaire de "l'échantillon rural". Autant que cela était possible, les mêmes districts ont été choisis pour la période

18. Le texte parle de "boroughs" (N. d. T.).

19. Le texte cite les "Boundary Commissioners". (pp 1888 (360 li, p. 1) : "Local Government Boundaries Commission. Report of the Boundary Commissioners of England and Wales 1888. Vol. 1". Je suis redevable à Pauline Round pour l'aide qu'elle $m$ 'a apportée pour la réunion et la réalisation des cartes. J'ai aussi utilisé la compilation des changements dans les districts d'enregistrement présentée dans l'appendice 1 de la thèse de Naomi Williams ("Ph. D").

20. Étant donné les difficultés que présenterait ici une traduction, j'ai préféré garder l'anglais (N. d. T.).

21. L'échantillon rural comprend : Farnborough, Godstone, Hoo, Hollingbourn, Cranbrook, Teneterden, East Ashford, Bridge, Hailsham, Uckfiled, Thakeham, Alton, Kingsclere, Hungerford, Faringdon, Wantage, Royston, Winslow, Ampthill, Caxton, Chesterton, Ely, North Witchford, Ongar, Dunmow, Risbridge. Thingoe, Hartismere, Hoxne, Bosmere, Samford, Flegg, Tunstead, Henstead, Loddon, Wayland, Pewsey, Amesbury, Wilton, South Molton, Holsworthy, Stratton, Northleach, Winchcomb, Weobly, Bromyard, Clun, Market Drayton, Holbeach, Garstang, Pocklington, Skirlaugh, Easingwold, Helmsley, Pickering, Rothbury, Longtown, Bootle, Builth, Knighton, Rhayader, Llanfyllin, Bala, Pwlheli. Farnborough est exclue depuis la décennie 1891-1901 car elle a été absorbée dans une autre unité, moins agricole. 
1891-1901. La carte I donne la localisation géographique des échantillons urbain et rural.

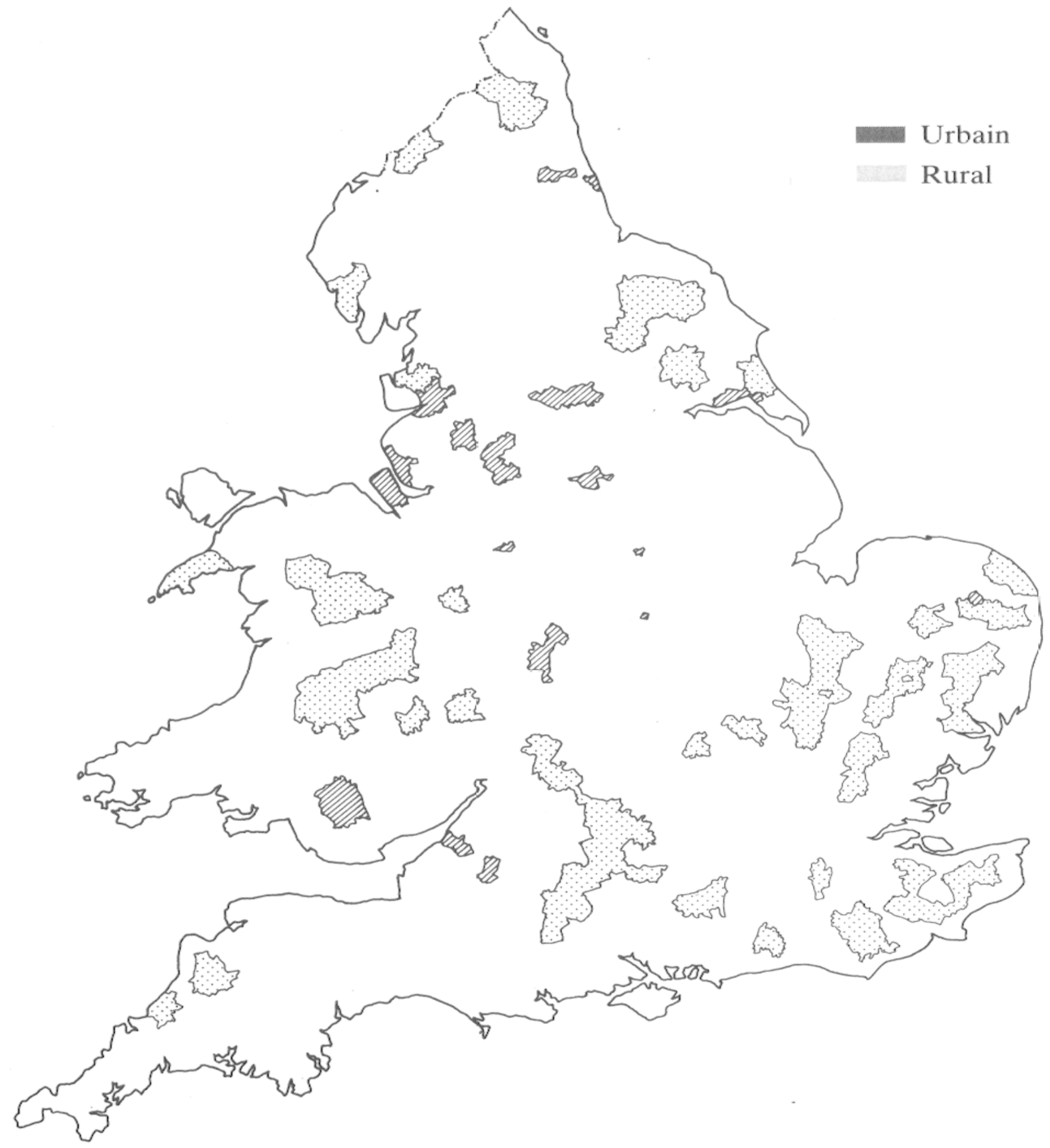

Carte I. - Districts urbains et ruraux d'enregistrement 1851-1900. 


\section{Définir “la population à risque".}

Les suppléments décennaux publiés par le Bureau de l'état civil donnent les chiffres de décès par district en tenant compte de l'âge, du sexe et des causes de décès en 1851-1860, et de manière séparée à la fois l'âge et le sexe, les causes de décès et le sexe pour 1891-1900. Les catégories utilisées en 1851-1860 sont d'abord chaque année jusqu'à cinq ans, puis les groupes d'âges de cinq ans de 5 ans à 25 , les groupes de dix ans de 25 à 85 ; quant aux défunts âgés de plus de 84 ans, ils constituent une seule et même catégorie. On trouve les mêmes groupes d'âges en 1891-1900, à la seule exception de la dernière catégorie qui commence cette fois à 75 ans.

En 1891-1900, nous avons aussi le nombre des mariages et celui des naissances par sexe. Pour chaque district d'enregistrement, les naissances des années 18511860 sont données dans le rapport annuel publié pour l'année par le Bureau de l'état civil. La population à risque, qui correspond à la première année de vie, peut donc être définie par le nombre des naissances enregistrées. Les populations à risque d'un autre âge et les groupes par sexes doivent être reconstruits à partir des volumes publiés pour les recensements décennaux. À cet endroit, nous avons intercalé les populations dans les groupes par âges et par sexes entre les recensements en partant de l'idée que le pourcentage annuel composé de changement des populations dans chaque groupe d'âges ou de sexes au cours des dix années qui séparent les recensements, est constant quoique différent ${ }^{22}$.

Cette procédure soulève un certain nombre de difficultés. La première est que les volumes du recensement de 1851 n'ont établi aucune différenciation à l'intérieur du groupe d'âges de 0 à 4 ans ; or, comme la mortalité infantile a pour nous une importance particulière, nous ne pouvons pas utiliser ces données d'ensemble. Néanmoins, les volumes du recensement de 1861 prenant en compte chaque groupe annuel d'âges, en dessous de cinq ans, nous avons décidé de travailler avec les groupes 0 à 1 an et 1 an à 4 ans. Il y a deux manières d'estimer la population qui compose ce deuxième groupe. Si l'on prend la population entre 0 et 5 ans pour chaque décennie, on peut soustraire les naissances et ajouter les décès infantiles puisque les naissances moins les décès infantiles donnent une approximation acceptable du nombre d'enfants vivant dans la première année à chaque époque. Alternativement, on peut prendre le groupe 0-4 ans et utiliser le rapport connu entre les groupes 0-4 et 1-4 ans en 1861 pour estimer la population interrecensement du groupe 1 à 4 . Les deux méthodes donnent des résultats tout à fait satisfaisants. Une analyse de quelques données tirées des recensements, de 1891 et 1901 pour lesquels les données ont été reportées par années d'âge en dessous de 5 ans, suggère que la dernière méthode ("par ensemble") est meilleure mais de façon marginale. Les deux méthodes ont été utilisées pour l'Angleterre, le pays de Galles, Londres, et un ensemble de districts d'enregistrement à la fois urbains et ruraux se situant dans le Nord-Ouest. Les résultats sont fournis dans le tableau 4.

22. Le taux de croissance d'une population entre deux recensements décennaux peut être calculé de la manière suivante :

$-\mathbf{R}-(\mathrm{P} 11-\mathrm{P} 1)^{-10}$

- pour l'époque choisie $(1+n)$

$\mathbf{P}(\mathbf{1}+\mathrm{n})=\mathrm{P} 1^{\times} \mathbf{R}^{\mathrm{n}}$

- pour toute la période intercensitaire, la population cumulée est :

Total de la population intercensitaire $=P 1^{\times}\left(R^{10-1}\right)(R-1)$. 
Tableau 4. - Estimation de la population âgée de 1 à 4 ans en 1891-1900.

\begin{tabular}{|c|c|c|c|c|c|}
\hline & \multirow[t]{2}{*}{$\begin{array}{l}\text { Chiffres du } \\
\text { recensement }\end{array}$} & \multicolumn{2}{|c|}{$\begin{array}{c}\text { Calcul à partir } \\
\text { des naissances } \\
\text { et décès }\end{array}$} & \multicolumn{2}{|c|}{$\begin{array}{l}\text { Calcul à partir de la } \\
\text { population âgée } \\
\text { de } 1 \text { à } 4 \text { ans en } 1901\end{array}$} \\
\hline & & Nombre & $\begin{array}{l}\% \text { de } \\
\text { recensement }\end{array}$ & Nombre & $\begin{array}{l}\% \text { de } \\
\text { recensement }\end{array}$ \\
\hline $\begin{array}{l}\text { Angleterre et } \\
\text { pays de Galles }\end{array}$ & 28529597 & 28511901 & 0.999 & 28489014 & 0.999 \\
\hline Londres & 3896643 & 3870597 & 0.993 & 3884628 & 0.997 \\
\hline \multicolumn{6}{|l|}{$\begin{array}{l}\text { Districts } \\
\text { d'enregistrement }\end{array}$} \\
\hline 451. Wirral & 37006 & 37187 & 1.005 & 37079 & 1.002 \\
\hline 452. Birkenhead & 137734 & 138483 & 1.005 & 137176 & 0.996 \\
\hline 453. Liverpool & 127518 & 125770 & 0.986 & 127275 & 0.998 \\
\hline 454. Toxteth Park & 123015 & 124059 & 1.008 & 122177 & 0.993 \\
\hline 455. West Derby & 484567 & 488499 & 1.008 & 481443 & 0.994 \\
\hline 456. Prescot & 158282 & 157470 & 0.995 & 158484 & 1.001 \\
\hline 457. Ormskirk & 86288 & 85845 & 0.995 & 86719 & 1.005 \\
\hline
\end{tabular}

La deuxième difficulté a pour origine les changements survenus dans les limites des districts d'enregistrements entre deux recensements. Les naissances, mariages et décès relevés par le Bureau de l'état civil subissent à des périodes différentes des modifications. Pour la mortalité infantile, ce n'est guère un problème puisque les naissances comme les décès infantiles correspondent à la même unité même si celle-ci peut changer au cours du temps. Pour les autres groupes d'âges, au contraire, il est possible qu'en intercalant des populations entre recensements, nous commettions l'erreur de compter des ensembles correspondant à des unités différentes. Néanmoins, nous savons à quelle date précise ont lieu les changements et les chiffres des populations concernées pour chaque recensement.

Par exemple, le district d'enregistrement d'Holsworthy ( $\mathrm{n}^{\circ} 298$ ), qui fait partie de notre échantillon rural, a été modifié entre les recensements de 1851 et de 1861 . Le Bureau de l'état civil nous apprend dans son rapport de 1861 que "la paroisse de Bradworthy (1 071 habitants en 1851 et 981 en 1861) a été transférée du sousdistrict de Bideford ( $\left.n^{\circ} 297\right)$ auquel elle appartenait à celui de Milton Damerel, s'ajouter sous-district de Holsworthy ( $\left.n^{\circ} 298\right)$, le 13 juillet $1859^{\prime \prime}$; c'est donc seulement pour la période qui va de cette date à celle du 31 décembre 1860 mais non pour la majeure partie de la décennie que les actes de Bradworthy sont venus s'ajouter à ceux du district de Holsworthy : différents moyens nous permettent de mesurer ce que cela représente : en l'espèce $10 \%$ de notre population à risque pour une année et demie. Dans une note suivante, le Bureau de l'état civil nous apprend qu'un changement est intervenu dans la définition des districts de Holsworthy et de Launceston $\left(\mathrm{n}^{\circ} 301\right)$ par le transfert des paroisses de Broadwoodwiger, St. Giles-in-the-Heath, Virginstone et du "hundred" de Northcott (soit 1532 habitants en 1851 et 1425 en 1861) dans lc sous-district de St.-Stephen qui appartient à Launceston, en avril 1853. Dans ce dernier cas, la perte de population est de l'ordre de $15 \%$ pour 7 années et deux tiers de la décennie. 
Enfin, lorsque nous voulons établir la population entre les recensements, nous constatons que les limites entre les groupes par sexes sont différemment constituées dans le district de Holsworthy en 1851 et 1861 . Néanmoins, nous pouvons changer de manière proportionnelle la composition par sexe et par âge de la population de 1861 pour obtenir des chiffres qui donnent une estimation de ce qui se serait passé s'il n'y avait eu aucun changement de limites. C'est ainsi qu'en 1861 la population du nouveau district de Holsworthy était de 9876 habitants ; s'il n'y avait eu aucun changement de limites, elle aurait été de 10320 , soit $4,5 \%$ en plus. Si nous ajoutons $4,5 \%$ aux groupes par sexe et par âge en 1861 , nous pouvons parfaitement travailler sur la composition par sexes et par âges entre les recensements en partant de l'idée que tout se passe comme s'il n'y avait pas eu de changements de limites du district. S'il n'y avait eu aucune correction, la population entre les recensements aurait été de 106867 personnes alors qu'en les faisant elle a été de 108955 . À partir de ces données, nous pouvons faire les ajustements nécessaires : ils nous amènent à soustraire 9792 personnes de notre estimation non corrigée de 108955 personnes; nous arrivons ainsi à 99161 personnes, soit une réduction de $7,2 \%$, ce qui entraîne une augmentation du taux de mortalité puisqu'il correspond à une population plus faible. En réduisant ainsi notre population à risque nous augmentons proportionnellement nos estimations de mortalité, avec pour conséquence une chute dans les tableaux d'espérance de vie. De ce fait, notre estimation de l'espérance de vie à la naissance tombe de 45,66 années à 44,55 .

Le tableau 5 montre à la fois les totaux de population concernés par les changements de limites et leurs effets sur le calcul des populations entre deux recensements. Hunslet est inclus pour 1851-1860 puisque le changement de limites survient avec le recensement de 1861 , et bien qu'il se situe après la période couverte pour les naissances et décès. Pour les zones urbaines, les pourcentages de changements des populations enregistrées se rapportent à l'unité urbaine tout entière bien que le district d'enregistrement concerné n'en soit qu'une partie. Les changements entre districts contenus dans la même unité urbaine ne sont pas reportés sur le tableau puisqu'ils n'ont pas d'effets sur nos estimations de population à risque, même si le véritable chiffre des changements survenant dans les années 1890 doit être pris en compte. Il faut également le faire pour le volume des changements et pour la redéfinition continuelle des districts à l'intérieur desquels se situent nos unités urbaines, et donc souligner la nécessité de consulter sans cesse des cartes lorsque nous utilisons les données établies à partir des districts d'enregistrement. 
Tableau 5. - Les effets des changements de limites sur la population des districts urbains et ruraux.

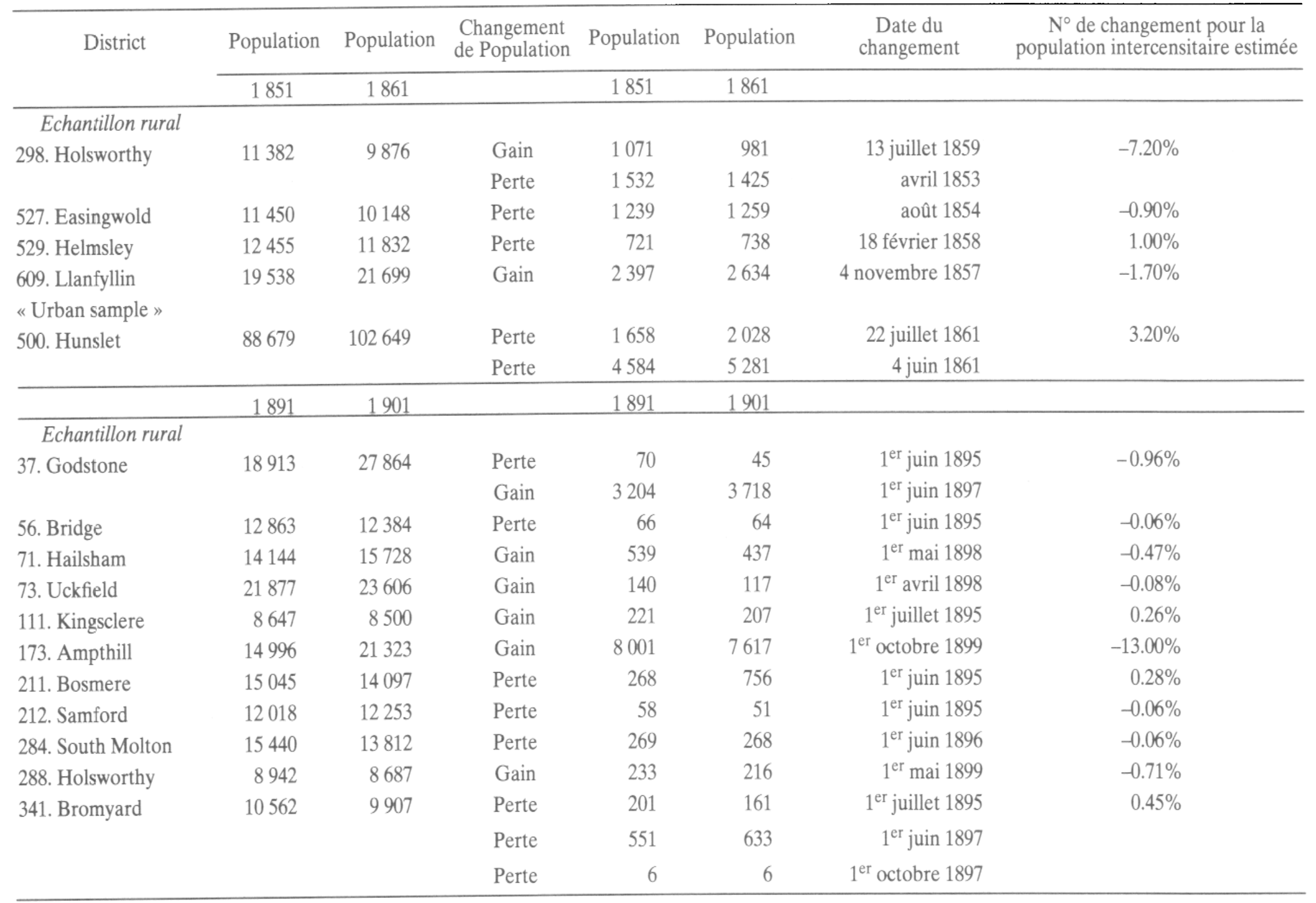




\begin{tabular}{|c|c|c|c|c|c|c|c|}
\hline District & Population & Population & $\begin{array}{l}\text { Changement } \\
\text { de Population }\end{array}$ & Population & Population & $\begin{array}{l}\text { Date du } \\
\text { changement }\end{array}$ & $\begin{array}{l}\mathrm{N}^{\circ} \text { de changement pour la } \\
\text { population intercensitaire estimée }\end{array}$ \\
\hline \multirow[t]{2}{*}{ 416. Holbeach } & 17813 & 17612 & Perte & 0 & 12 & $1^{\mathrm{er}}$ novembre 1897 & $-0.17 \%$ \\
\hline & & & Gain & 46 & 50 & $1^{\mathrm{er}}$ novembre 1897 & \\
\hline 523. Skirlaugh & 9304 & 9359 & Perte & 34 & 30 & $1^{\mathrm{er}}$ juillet 1895 & $-0.04 \%$ \\
\hline 528. Easingwold & 8847 & 9909 & Gain & 1537 & 1308 & 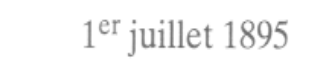 & $1.87 \%$ \\
\hline 607. Builth & 7834 & 8976 & Gain & 91 & 88 & $1^{\text {er juin } 1897}$ & $-0.01 \%$ \\
\hline \multicolumn{8}{|l|}{ Echantillon urbain } \\
\hline \multirow[t]{3}{*}{ 277. Plymouth } & 84253 & 107636 & Gain & 4657 & 9642 & $1^{\text {er }}$ janvier 1897 & $-1.47 \%$ \\
\hline & & & Gain & 0 & 25 & $1^{\mathrm{er}}$ mars 1898 & \\
\hline & & & Gain & 21 & 39 & $1^{\mathrm{er}}$ avril 1899 & \\
\hline 279. Devonport & 54803 & 70437 & Gain & 1178 & 6520 & 9 novembre 1898 & \\
\hline 320. Bristol & 55549 & 328945 & Gain & 330 & 3209 & $1^{\text {er }}$ janvier 1899 & $-0.27 \%$ \\
\hline 363. Stoke upon Trent & 121459 & 155422 & Gain & 13657 & 15773 & $1^{\text {er }}$ Mai 1896 & $0.33 \%$ \\
\hline 385. King's Norton & 12742 & 187087 & Perte & 64 & 61 & 9 novembre 1897 & $0.00 \%$ \\
\hline 394. Solihull & 29822 & 48618 & Gain & 105 & 104 & $1^{\mathrm{er}}$ novembre 1899 & \\
\hline 409. Leicester & 174624 & 211579 & Gain & 32579 & 60090 & $1^{\mathrm{er}}$ janvier 1892 & $7.13 \%$ \\
\hline \multirow[t]{2}{*}{ 431. Nottingham } & 177078 & 239743 & Gain & 287 & 345 & $1^{\mathrm{er}}$ juillet 1895 & $-5.13 \%$ \\
\hline & & & Gain & 36512 & 46411 & $1^{\mathrm{er}}$ janvier 1899 & \\
\hline 452. Birkenhead & 130591 & 165171 & Gain & 2951 & 2971 & $1^{\mathrm{er}}$ avril 1898 & $-0.05 \%$ \\
\hline 467. Prestwich & 149537 & 196832 & Gain & 2476 & 6970 & $1^{\text {er }}$ juillet 1895 & $0.33 \%$ \\
\hline 469. Oldham & 201153 & 215624 & Gain & 855 & 1120 & $1^{\text {er }}$ juillet 1895 & $-0.02 \%$ \\
\hline \multirow[t]{2}{*}{ 497b. North Bierley } & 138906 & 129067 & Perte & 2777 & 2603 & $1^{\text {er }}$ juin 1895 & $-0.06 \%$ \\
\hline & & & Perte & 436 & 389 & 9 novembre 1899 & \\
\hline 588. Merthyr Tydfil & 117205 & 135540 & Perte & 1562 & 3039 & $1^{\text {er juin } 1895}$ & $-0.06 \%$ \\
\hline
\end{tabular}




\section{Les différences dans les changements de la mortalité entre les districts urbains et ruraux.}

\section{Les modèles de mortalité dans l'échantillon rural.}

Les catégories de maladies utilisées ici reprennent celles employées par McKeown et Record ; elles ont été plus complètement expliquées dans une autre publication $^{23}$. Le tableau 6 indique qu'au cours de la décennie 1851-1860 l'espérance de vie à la naissance pour les hommes était dans nos districts ruraux de 46,9 années et de 46,76 seulement pour les femmes. À l'âge de 20 ans, les chiffres respectifs étaient de 43,45 et 42,04 . Un demi-siècle plus tard le progrès est réel puisque pour les années 1891 à 1900 leurs espérances de vie à la naissance sont de 52,83 et 54,89 . Désormais donc les femmes ont une meilleure espérance de vie, ce que l'on retrouve à 20 ans : 45,91 pour elles contre 45,28 pour les hommes. À certains égards, il semble que cette plus forte mortalité féminine rurale soit une anomalie sur laquelle il conviendra de mener des recherches plus approfondies. Pour ce qui nous concerne actuellement, il faut mettre en valeur que le déclin de la mortalité n'a pas seulement concerné, dans la deuxième moitié du XIXe siècle, les villes en train de rattraper les campagnes : les progrès furent également très significatifs pour celles-ci.

Tableau 6. - Tables de mortalité pour les districts ruraux 1851-1860, 1891-1900.

\begin{tabular}{|c|c|c|c|c|c|c|c|c|c|c|c|c|}
\hline & 0 & $1-4$ & $5-9$ & $10-14$ & $15-19$ & $20-24$ & $25-34$ & $35-44$ & $45-54$ & $55-64$ & $65-74$ & $>74$ \\
\hline \multicolumn{13}{|c|}{ Districts ruraux 1851-1860, hommes } \\
\hline${ }_{n} m_{x}$ & 0.1444 & 0.0212 & 0.0069 & 0.0043 & 0.0054 & 0.0082 & 0.0079 & 0.0087 & 0.0119 & 0.0220 & 0.0546 & 0.1578 \\
\hline$e_{x}$ & 46.90 & 53.77 & 54.35 & 51.16 & 47.22 & 43.45 & 40.16 & 33.06 & 25.60 & 18.21 & 11.48 & 6.34 \\
\hline
\end{tabular}

Districts ruraux 1851-1860, femmes

$\begin{array}{llllllllllllll}{ }_{\mathrm{n}} \mathrm{m}_{\mathrm{x}} & 0.1162 & 0.0205 & 0.0071 & 0.0055 & 0.0084 & 0.0101 & 0.0101 & 0.0108 & 0.0123 & 0.0221 & 0.0513 & 0.1491\end{array}$

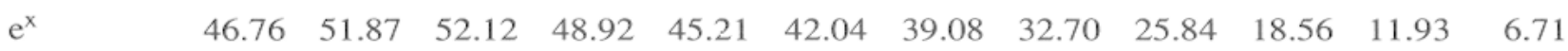

Districts ruraux 1851-1860, total

$\begin{array}{llllllllllllll}{ }_{\mathrm{n}} \mathrm{m}_{\mathrm{x}} & 0.1307 & 0,0208 & 0.0070 & 0.0049 & 0.0068 & 0.0091 & 0.0090 & 0.0097 & 0.0121 & 0.0220 & 0.0529 & 0.1532\end{array}$

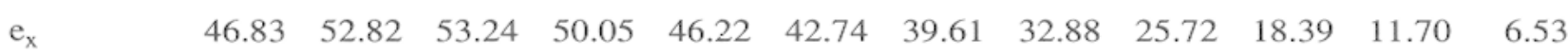

Districts ruraux 1891-1990, hommes

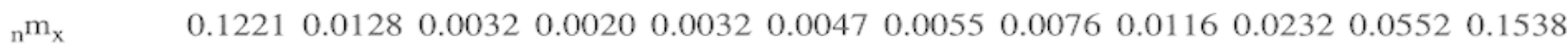

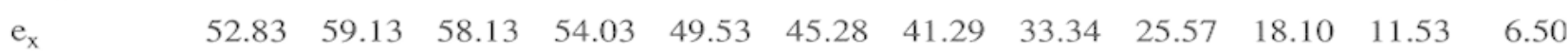

Districts ruraux 1891-1990, femmes

$\begin{array}{lllllllllllll}{ }_{\mathrm{n}} \mathrm{m}_{\mathrm{x}} & 0.0940 & 0.0120 & 0.0032 & 0.0024 & 0.0042 & 0.0051 & 0.0057 & 0.0076 & 0.0104 & 0.0212 & 0.0512 & 0.1421\end{array}$

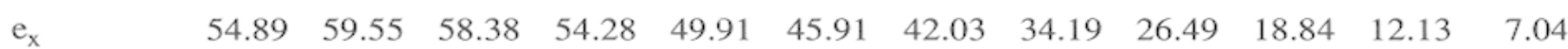

Districts ruraux 1891-1900, total

$\begin{array}{lllllllllllll}{ }_{\mathrm{n}} \mathrm{m}_{\mathrm{x}} & 0.1083 & 0.0124 & 0.0032 & 0.0022 & 0.0036 & 0.0049 & 0.0056 & 0.0076 & 0.0110 & 0.0222 & 0.0532 & 0.1477\end{array}$

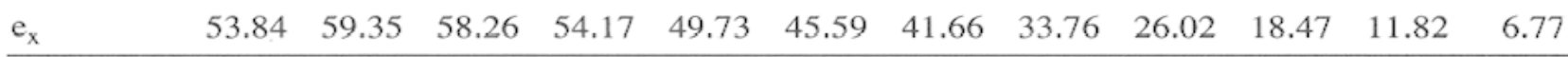

23. G. KEARNS, "The urban penalty and the population history of England", art. cité. 
Tableau 7. - La contribution des différentes maladies aux changements dans l'espérance de vie dans les districts ruraux.

\begin{tabular}{|c|c|c|c|c|c|c|c|c|c|c|c|c|}
\hline Zones rurales & 0 & $1-4$ & $5-9$ & $10-14$ & $15-19$ & $20-24$ & $25-34$ & $35-44$ & $45-54$ & $55-64$ & $65-74$ & $>74$ \\
\hline Tuberculose respiratoire & 2.274 & 2.453 & 2.525 & 2.497 & 2.381 & 2.061 & 1.561 & 0.809 & 0.441 & 0.206 & 0.057 & 0.005 \\
\hline Autres tuberculoses & 0.218 & 0.183 & 0.069 & 0.026 & 0.012 & 0.007 & 0.014 & 0.016 & 0.032 & 0.023 & 0.012 & 0.002 \\
\hline Typhus & 1.128 & 1.261 & 1.196 & 1.047 & 0.904 & 0.728 & 0.592 & 0.417 & 0.299 & 0.195 & 0.110 & 0.047 \\
\hline Scarlatine & 0.885 & 0.952 & 0.532 & 0.198 & 0.077 & 0.048 & 0.030 & 0.017 & 0.007 & 0.004 & 0.003 & 0.001 \\
\hline Diarrhées & 0.323 & 0.326 & 0.235 & 0.219 & 0.213 & 0.207 & 0.200 & 0.179 & 0.163 & 0.143 & 0.117 & 0.088 \\
\hline Total (1) & 5.050 & 5.404 & 4.723 & 4.109 & 3.687 & 3.127 & 2.451 & 1.469 & 0.961 & 0.581 & 0.303 & 0.145 \\
\hline Variole & 0.102 & 0.087 & 0.070 & 0.059 & 0.054 & 0.048 & 0.037 & 0.022 & 0.011 & 0.005 & 0.002 & 0.001 \\
\hline Coqueluche & 0.028 & 0.058 & 0.023 & 0.002 & 0.001 & 0.001 & 0.000 & 0.000 & 0.000 & 0.000 & 0.000 & 0.000 \\
\hline Rougeole & 0.032 & 0.056 & 0.015 & 0.000 & -0.001 & 0.000 & -0.003 & -0.001 & 0.001 & 0.000 & 0.000 & 0.000 \\
\hline Diphthérie & -0.145 & -0.166 & -0.088 & -0.011 & -0.001 & -0.002 & -0.001 & -0.001 & -0.001 & -0.002 & 0.000 & 0.001 \\
\hline Total (2) & 0.017 & 0.034 & 0.020 & 0.051 & 0.053 & 0.047 & 0.034 & 0.020 & 0.011 & 0.004 & 0.002 & 0.001 \\
\hline Maladies cérébrales & 0.497 & 0.097 & -0.018 & -0.062 & -0.104 & -0.130 & -0.163 & -0.205 & -0.210 & -0.201 & -0.162 & -0.180 \\
\hline Maladies cardiaques & -0.209 & -0.245 & -0.291 & -0.315 & -0.333 & -0.356 & -0.382 & -0.454 & -0.517 & -0.527 & -0.485 & -0.386 \\
\hline Maladies pulmonaires & -0.197 & -0.021 & 0.013 & -0.008 & -0.033 & -0.055 & -0.096 & -0.152 & -0.197 & -0.228 & -0.288 & -0.375 \\
\hline Maladies stomacales & -0.043 & 0.254 & 0.349 & 0.351 & 0.345 & 0.338 & 0.318 & 0.275 & 0.223 & 0.146 & 0.047 & -0.034 \\
\hline Maladies rénales & -0.199 & -0.217 & -0.228 & -0.233 & -0.236 & -0.237 & -0.243 & -0.246 & -0.236 & -0.203 & -0.153 & -0.115 \\
\hline Total (3) & -0.114 & -0.091 & -0.131 & -0.222 & -0.314 & -0.390 & -0.512 & -0.722 & -0.868 & -0.933 & -0.946 & -0.974 \\
\hline Cancers & -0.413 & -0.475 & -0.514 & -0.529 & -0.541 & -0.560 & -0.585 & -0.626 & -0.631 & -0.527 & -0.336 & -0.168 \\
\hline Maladies infectueuses & 1.082 & 0.889 & 0.595 & 0.484 & 0.452 & 0.435 & 0.415 & 0.382 & 0.328 & $0^{\circ} .272$ & 0.212 & 0.145 \\
\hline Maladies héréditaires & 0.014 & 0.016 & 0.018 & 0.019 & 0.019 & 0.021 & 0.021 & 0.020 & 0.007 & 0.003 & -0.004 & -0.001 \\
\hline Maladies articulaires & 0.078 & 0.086 & 0.087 & 0.080 & 0.069 & 0.057 & 0.048 & 0.034 & 0.027 & 0.018 & 0.010 & 0.002 \\
\hline Maladies de la peau & 0.066 & 0.050 & 0.044 & 0.042 & 0.040 & 0.039 & 0.038 & 0.032 & 0.029 & 0.025 & 0.019 & 0.010 \\
\hline $\begin{array}{l}\text { Morts lors des } \\
\text { accouchements }\end{array}$ & 0.001 & 0.001 & 0.001 & 0.001 & 0.001 & -0.004 & 0.001 & 0.017 & 0.001 & 0.000 & 0.000 & 0.000 \\
\hline Morts violentes & 0.048 & 0.091 & 0.015 & -0.034 & -0.077 & 0.083 & -0.083 & -0.072 & -0.065 & -0.047 & -0.036 & -0.026 \\
\hline Autres & 1.169 & 0.620 & 0.368 & 0.353 & 0.377 & 0.434 & 0.498 & 0.631 & 0.821 & 1.027 & 1.291 & 1.596 \\
\hline Total (4) & 2.025 & 1.246 & 0.574 & 0.372 & 0.295 & 0.294 & 0.306 & 0.364 & 0.458 & 0.712 & 1.106 & 1.527 \\
\hline Toutes causes de décès & 7.014 & 6.526 & 5.024 & 4.118 & 3.512 & 2.857 & 2.045 & 0.883 & 0.299 & 0.078 & 0.123 & 0.244 \\
\hline
\end{tabular}


On ne peut décomposer cette amélioration des taux de mortalité par causes ou par sexes car les notes fournies par le Bureau de l'état civil dans son supplément décennal de 1891-1900 ne donnent aucune information à cet égard au niveau des districts. Cependant, les progrès les plus importants s'expliquent, comme pour le tableau 8, par la chute de la mortalité entraînée par la tuberculose respiratoire : on lui doit un progrès de 2,27 années dans l'espérance de vie ; ce progrès concerne essentiellement les adultes car le gain survenu après 20 ans représente 2,06 années. À l'opposé, plus de la moitié des progrès concernant les autres maladies infectieuses ("zymotic" dans le texte anglais) qui furent de 1,08 années, et celui concernant la variole, qui fut de 0,10 année, se situèrent avant l'âge de 20 ans ; quant à celui de 0,89 année dû à la chute des effets de la scarlatine, il se situa au cours des 10 premières années de vie. Une faible amélioration : 0,32 année, vint d'un recul des maladies diarrhéiques (en incluant dans celles-ci le choléra et la dysenterie). Le groupe des "typhus", qui inclut la typhoïde et les fièvres "continues", peut, comme la diarrhée, être considéré principalement comme un ensemble de maladies dues à "la saleté"; ici, on a eu un progrès de 1,13 années, ce qui représente une partie importante du recul d'ensemble de la mortalité.

Tableau 8. - Tables de mortalité pour les districts urbains.

\begin{tabular}{llllll}
\hline 0 & $1-4$ & $5-9$ & $10-14$ & $15-1920-2425-3435-4445-5455-6465-74 \quad>74$ \\
\hline
\end{tabular}

Grandes villes 1851-1860, hommes

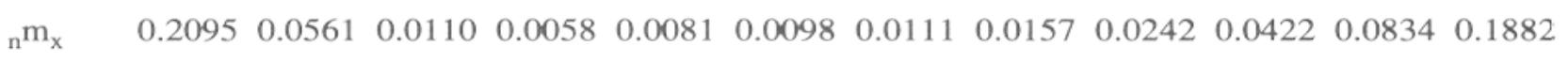
$\begin{array}{lllllllllllll}\mathrm{e}_{\mathrm{x}} & 32.86 & 40.49 & 46.22 & 43.70 & 39.92 & 36.45 & 33.15 & 26.47 & 20.12 & 14.28 & 9.24 & 5.31\end{array}$

Grandes villes 1851-1860, femmes

$\begin{array}{lllllllllllll}{ }_{\mathrm{n}} \mathrm{m}_{\mathrm{x}} & 0.1740 & 0.0553 & 0.0108 & 0.0054 & 0.0076 & 0.0086 & 0.0110 & 0.0144 & 0.0194 & 0.0340 & 0.0708 & 0.1707\end{array}$

$\begin{array}{lllllllllllll}\mathrm{e}_{\mathrm{x}} & 35.71 & 42.17 & 48.16 & 45.70 & 41.88 & 38.40 & 34.98 & 28.47 & 22.10 & 15.77 & 10.18 & 5.86\end{array}$

Grandes villes $1851-1860$, total

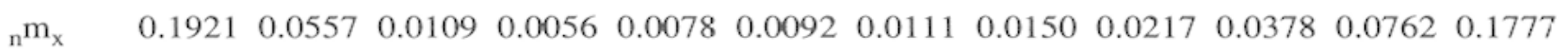

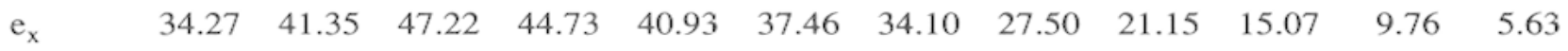

Grandes villes 1891-1900, hommes

$\begin{array}{lllllllllllll}{ }_{\mathrm{n}} \mathrm{m}_{\mathrm{x}} & 0.1945 & 0.0316 & 0.0049 & 0.0027 & 0.0043 & 0.0055 & 0.0077 & 0.0139 & 0.0234 & 0.0433 & 0.0830 & 0.1697\end{array}$

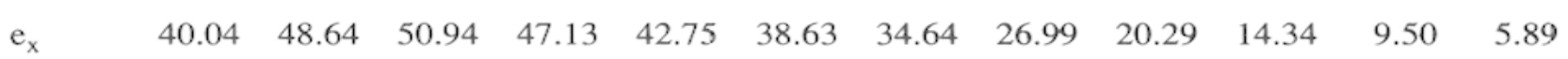

Grandes villes 1891-1900, femmes

$\begin{array}{lllllllllllll}{ }_{\mathrm{n}} \mathrm{m}_{\mathrm{x}} & 0.1629 & 0.0305 & 0.0049 & 0.0027 & 0.0038 & 0.0047 & 0.0068 & 0.0116 & 0.0183 & 0.0347 & 0.0700 & 0.1535\end{array}$

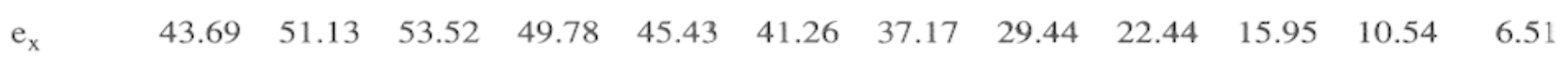

Grandes villes 1891-1900, total

$\begin{array}{lllllllllllll}{ }_{\mathrm{n}} \mathrm{m}_{\mathrm{x}} & 0.1789 & 0.0311 & 0.0049 & 0.0027 & 0.0041 & 0.0050 & 0.0072 & 0.0127 & 0.0208 & 0.0386 & 0.0756 & 0.1598\end{array}$

\begin{tabular}{lllllllllllll}
$\mathrm{e}_{\mathrm{x}}$ & 41.85 & 49.91 & 52.26 & 48.49 & 44.13 & 39.98 & 35.94 & 28.25 & 21.41 & 15.21 & 10.08 & 6.26 \\
\hline
\end{tabular} 


\section{Les modèles de mortalité dans l'échantillon urbain.}

Dans la décennie 1851-1860, l'espérance de vie était nettement plus basse en ville qu'à la campagne :

\begin{tabular}{ccccc}
\hline & \multicolumn{2}{c}{ Ville } & \multicolumn{2}{c}{ Campagne } \\
\hline & $\mathrm{H}$ & $\mathrm{F}$ & $\mathrm{H}$ & $\mathrm{F}$ \\
$\begin{array}{c}\text { Espérance de vie } \\
\text { à la naissance }\end{array}$ & 32,86 & 35,71 & 46,90 & 46,76 \\
$\begin{array}{c}\text { Espérance de vie à 20 ans } \\
\text { (voir tableau 8) }\end{array}$ & 35,45 & 38,40 & 43,45 & 42,05 \\
\hline
\end{tabular}

L'écart était particulièrement marqué pour les hommes. D'où l'importance des progrès survenus dans la deuxième moitié du siècle : entre 1891 et 1900 , l'espérance de vie à la naissance des hommes s'est accrue de 7 années et celle des femmes d'un peu plus de 8, ce qui a encore augmenté l'écart : elle est alors respectivement de 40,04 et 43,69 années. L'amélioration générale, en regroupant les deux sexes, a été de 7,59 années : les deux tiers de ces progrès se sont situés avant la vingtième année. Au-delà de 20 ans, elle a été de 2,52 années mais plus marquée pour les hommes : 3,41 années, que pour les femmes : 2,86.

Ce progrès urbain de 7,59 années n'a réduit que de manière marginale l'avantage des campagnes puisque celles-ci ont connu un progrès de 7,01 années de l'espérance de vie. Mais les causes des changements furent sans doute différentes puisque les causes médicales de l'amélioration de l'espérance de vie le furent. Les profils des mortalités urbaine et rurale n'ont pu être décrits que de manière très générale jusqu'ici. Cependant, pour la décennie 1851-1860, nous pouvons préciser l'interaction des facteurs que sont l'âge, le sexe et la cause du décès, comme le montrent les tableaux 9 et 10 , pour lesquels le chiffre 100 indique une égalité complète, les blancs indiquant que tous les facteurs retenus ne jouent pas de rôle.

Pour les hommes adultes, la différence essentielle entre les villes et les campagnes concerne la tuberculose respiratoire, et plus particulièrement les maladies pulmonaires. D'autres causes de décès qui frappaient plus lourdement les hommes dans les villes faisaient également disparaître les femmes, telles les diarrhées. Étant donné ce que nous savons sur les conditions alimentaires des familles appartenant à la classe ouvrière au XIXe siècle, il est impensable que ces différences dans la mortalité par tuberculose respiratoire proviennent de différences réelles dans l'alimentation des hommes et des femmes en ville et à la campagne. Il paraît plus utile de rechercher une explication du côté de la façon de se nourrir, c'est-à-dire de nous demander dans quelle mesure le manque de nourriture ou une ivrognerie plus forte ont pu jouer un grand rôle dans ces différences. Nous pouvons aussi nous demander s'il ne faut pas se tourner vers des maladies de surpeuplement qui se seraient transmises beaucoup plus rapidement et avec une force plus grande parmi les hommes qui s'entassaient dans les pensions, les taux de mortalité dus à ces maladies reculant lorsqu'ils les quittaient pour les appartements familiaux. Pour les femmes, ce qu'il faudra approfondir, c'est la différence qui existe quant aux effets de la variole en ville et à la campagne ; on a en effet à tous les âges un surplus massif de décès féminins dans les villes. Pour les enfants, ceci peut refléter une nourriturc urbainc plus pauvre des femmes, et parmi les adultes ce peut être une conséquence d'une plus grande vulnérabilité des mères qui allaitent aux maladies de leurs enfants. 
Tableau 9. - Le rapport entre les zones urbaines et les zones rurales pour la mortalité masculine 1851-1860. Comparaison des mortalités masculines.

\begin{tabular}{|c|c|c|c|c|c|c|c|c|c|c|c|c|}
\hline Zones rurales & 0 & $1-4$ & $5-9$ & $10-14$ & $15-19$ & $20-24$ & $25-34$ & $35-44$ & $45-54$ & $55-64$ & $65-74$ & $>74$ \\
\hline Tuberculose respiratoire & 61.5 & 168.0 & 123.0 & 142.0 & 153.8 & 101.2 & 117.2 & 174.0 & 202.0 & 172.4 & 141.4 & 217.9 \\
\hline Autres tuberculoses & 219.3 & 310.2 & 148.5 & 149.0 & 137.4 & 148.5 & 145.4 & 193.1 & 163.5 & 180.2 & 133.4 & 98.0 \\
\hline Typhus & 219.7 & 279.9 & 148.0 & 119.2 & 106.6 & 90.1 & 102.1 & 123.6 & 131.8 & 134.6 & 156.9 & 181.4 \\
\hline Scarlatine & 191.3 & 278.3 & 184.7 & 93.7 & 65.7 & 43.2 & 72.4 & 65.1 & 67.7 & 122.3 & 49.4 & 0.0 \\
\hline Diarrhées & 380.9 & 501.0 & 298.4 & 304.8 & 257.8 & 312.1 & 265.1 & 290.7 & 309.1 & 315.2 & 273.2 & 231.9 \\
\hline Total (1) & 251.9 & 302.0 & 164.6 & 128.8 & 137.6 & 102.8 & 119.3 & 169.4 & 192.4 & 181.4 & 181.8 & 215.4 \\
\hline Variole & 370.8 & 1300.2 & 1004.9 & 238.7 & 305.6 & 164.5 & 170.0 & 87.8 & 58.5 & 30.6 & 6.2 & 0.0 \\
\hline Coqueluche & 134.1 & 284.4 & 206.3 & 1929.8 & & 2439.1 & 2536.6 & & & & & \\
\hline Rougeole & 301.7 & 434.3 & 208.2 & 65.3 & 109.7 & 30.2 & 207.9 & & & & & \\
\hline Diphthérie & 107.6 & 72.6 & 35.1 & 28.0 & 15.2 & 16.6 & 31.2 & 36.0 & 55.8 & 45.1 & 65.9 & 140.0 \\
\hline Total (2) & 179.3 & 332.8 & 165.2 & 86.2 & 132.1 & 157.8 & 185.4 & 116.4 & 116.0 & 82.1 & 107.9 & 303.2 \\
\hline Maladies cérébrales & 194.5 & 221.6 & 160.3 & 118.7 & 121.0 & 122.4 & 140.6 & 190.4 & 208.8 & 214.8 & 164.7 & 132.3 \\
\hline Maladies cardiaques & 212.5 & 359.4 & 182.7 & 158.6 & 177.3 & 138.9 & 180.1 & 175.7 & 189.3 & 155.6 & 108.1 & 92.5 \\
\hline Maladies pulmonaires & 162.5 & 253.3 & 155.6 & 122.2 & 149.6 & 119.7 & 180.6 & 247.6 & 323.1 & 313.5 & 254.4 & 206.7 \\
\hline Maladies stomacales & 115.5 & 140.2 & 117.0 & 143.6 & 124.6 & 104.9 & 132.3 & 149.1 & 155.2 & 142.3 & 118.9 & 119.6 \\
\hline Maladies rénales & 66.3 & 235.8 & 222.4 & 264.9 & 208.2 & 184.4 & 149.6 & 184.1 & 161.6 & 160.2 & 101.7 & 119.2 \\
\hline Total (3) & 175.6 & 238.4 & 155.9 & 135.8 & 143.1 & 123.7 & 158.5 & 193.8 & 221.0 & 210.2 & 162.6 & 143.7 \\
\hline Cancers & 187.5 & 466.9 & 500.5 & 276.7 & 219.4 & 263.3 & 193.1 & 138.6 & 164.9 & 146.1 & 112.0 & 84.2 \\
\hline Maladies infectueuses & 105.7 & 153.1 & 84.0 & 85.8 & 138.4 & 104.2 & 158.9 & 177.1 & 175.7 & 143.0 & 106.5 & 81.3 \\
\hline Maladies héréditaires & 215.2 & & 166.8 & & & & & 54.0 & 390.3 & & 111.2 & 42.0 \\
\hline Maladies articulaires & 215.2 & 231.4 & 270.8 & 210.7 & 210.6 & 140.7 & 122.2 & 249.7 & 214.7 & 170.8 & 178.9 & 367.4 \\
\hline Maladies de la peau & 113.9 & 144.2 & 53.3 & 80.4 & 126.2 & 99.3 & 51.3 & 84.9 & 135.7 & 100.8 & 134.0 & 169.9 \\
\hline \multicolumn{13}{|c|}{ Morts lors de l'accouchement } \\
\hline Morts violentes & 275.0 & 151.0 & 192.0 & 129.6 & 176.5 & 170.7 & 171.4 & 165.7 & 200.9 & 188.7 & 192.6 & 328.1 \\
\hline Autres & 91.4 & 275.5 & 175.6 & 237.4 & 209.9 & 282.2 & 247.2 & 210.4 & 161.6 & 153.1 & 125.6 & 97.5 \\
\hline Total (4) & 96.2 & 211.0 & 151.4 & 160.4 & 192.6 & 192.8 & 191.2 & 182.2 & 181.2 & 159.3 & 128.9 & 100.9 \\
\hline Toutes causes de décès & 145.1 & 264.9 & 160.2 & 135.5 & 148.0 & 119.3 & 140.8 & 180.5 & 203.1 & 191.8 & 152.8 & 119.3 \\
\hline
\end{tabular}


Tableau 10. - Le rapport entre les zones urbaines et les zones rurales pour la mortalité feminine 1851 -1860.

Comparaison des mortalités féminines.

\begin{tabular}{|c|c|c|c|c|c|c|c|c|c|c|c|c|}
\hline Zones rurales & 0 & $1-4$ & $5-9$ & $10-14$ & $15-19$ & $20-24$ & $25-34$ & $35-44$ & $45-54$ & $55-64$ & $65-74$ & $>74$ \\
\hline Tuberculose respiratoire & 66.2 & 157.2 & 99.3 & 83.0 & 84.0 & 73.1 & 93.2 & 115.2 & 115.2 & 103.2 & 98.7 & 160.9 \\
\hline Autres tuberculoses & 161.7 & 265.4 & 132.1 & 98.0 & 81.5 & 75.9 & 120.3 & 158.2 & 128.6 & 95.0 & 80.0 & 58.6 \\
\hline Typhus & 204.9 & 268.8 & 167.2 & 95.4 & 77.2 & 72.1 & 85.8 & 103.3 & 115.5 & 136.0 & 132.9 & 177.5 \\
\hline Scarlatine & 189.6 & 290.5 & 174.4 & 74.3 & 64.7 & 44.2 & 50.7 & 33.6 & 39.2 & 80.4 & 10.9 & 25.6 \\
\hline Diarrhées & 442.8 & 486.8 & 302.8 & 237.7 & 233.0 & 231.9 & 296.8 & 372.7 & 326.1 & 313.1 & 281.9 & 216.6 \\
\hline Total (1) & 247.2 & 292.0 & 157.3 & 89.1 & 83.6 & 74.7 & 96.9 & 121.3 & 126.2 & 135.3 & 161.8 & 199.5 \\
\hline Variole & 1641.4 & 2326.9 & 1224.0 & 1543.0 & 711.1 & 807.5 & 944.7 & 1551.9 & 2503.7 & 4662.6 & 9780.2 & 7586.2 \\
\hline Coqueluche & 130.0 & 284.1 & 183.9' & 62.9 & 43.9 & & & & & & & \\
\hline Rougeole & 324.0 & 469.5 & 198.1 & 128.0 & 186.5 & 72.2 & 156.8 & 105.9 & 36.7 & & & \\
\hline Diphthérie & 115.2 & 101.5 & 50.3 & 27.9 & 16.3 & 22.9 & 29.8 & 24.4 & 31.5 & 48.2 & 76.3 & 51.3 \\
\hline Total (2) & 244.9 & 392.2 & 189.1 & 127.9 & 193.0 & 338.1 & 594.1 & 676.9 & 854.2 & 1584.3 & 6558.2 & 5074.6 \\
\hline Maladies cérébrales & 204.3 & 257.2 & 157.4 & 111.6 & 96.1 & 87.9 & 115.8 & 123.8 & 169.5 & 141.6 & 130.3 & 124.4 \\
\hline Maladies cardiaques & 487.1 & 400.3 & 215.8 & 164.1 & 111.5 & 126.2 & 126.2 & 143.9 & 145.6 & 113.5 & 91.3 & 79.0 \\
\hline Maladies pulmonaires & 165.1 & 266.7 & 140.9 & 106.2 & 112.5 & 111.6 & 145.8 & 231.6 & 387.5 & 331.8 & 246.8 & 216.7 \\
\hline Maladies stomacales & 147.1 & 200.9 & 107.0 & 92.6 & 89.8 & 93.7 & 125.6 & 164.4 & 153.7 & 140.3 & 124.2 & 142.8 \\
\hline Maladies rénales & 100.8 & 445.2 & 117.7 & 256.1 & 188.4 & 173.1 & 183.2 & 165.2 & 196.9 & 178.4 & 130.0 & 192.2 \\
\hline Total (3) & 188.8 & 263.0 & 146.9 & 118.7 & 103.9 & 105.0 & 130.8 & 163.7 & 198.1 & 172.1 & 146.1 & 140.9 \\
\hline Cancers & 875.2 & 868.1 & 1233.1 & 79.8 & 96.0 & 99.3 & 152.2 & 137.2 & 140.4 & 118.8 & 118.3 & 109.5 \\
\hline Maladies infectueuses & 124.8 & 158.5 & 93.0 & 84.0 & 123.1 & 102.1 & 170.7 & 132.0 & 137.2 & 110.6 & 83.5 & 82.3 \\
\hline Maladies héréditaires & 74.3 & 493.4 & & & 159.1 & 108.3 & 132.5 & 121.1 & 143.9 & 131.8 & 189.7 & 134.2 \\
\hline Maladies articulaires & 99.0 & 283.1 & 324.9 & 246.1 & 137.4 & 112.5 & 123.2 & 207.8 & 149.3 & 241.2 & 152.5 & 235.8 \\
\hline Maladies de la peau & 88.2 & 171.2 & 164.4 & 127.7 & 80.5 & 58.7 & 56.4 & 169.4 & 156.2 & 183.4 & 110.8 & 125.6 \\
\hline Morts lors de l'accouchement & & & & & 101.5 & 115.9 & 106.7 & 90.5 & 59.8 & & & \\
\hline Morts violentes & 191.1 & 129.7 & 145.3 & 128.0 & 131.7 & 196.1 & 219.9 & 188.5 & 216.6 & 211.0 & 222.9 & 195.8 \\
\hline Autres & 86.0 & 188.1 & 95.8 & 72.0 & 47.5 & 44.9 & 50.2 & 70.7 & 77.7 & 103.9 & 111.9 & 93.6 \\
\hline Total (4) & 91.9 & 171.6 & 116.6 & 99.8 & 92.0 & 98.5 & 105.6 & 107.4 & 121.2 & 117.6 & 113.9 & 95.4 \\
\hline Toutes causes de décès & 149.8 & 270.5 & 152.0 & 98.2 & 90.2 & 85.8 & 109.1 & 133.5 & 158.2 & 154.0 & 138.0 & 114.5 \\
\hline
\end{tabular}


Tableau 11. - La contribution des diverses maladies aux changements dans l'espérance de vie dans les zones urbaines

\begin{tabular}{|c|c|c|c|c|c|c|c|c|c|c|c|c|}
\hline Zones urbaines & 0 & $1-4$ & $5-9$ & $10-14$ & $15-19$ & $20-24$ & $25-34$ & $35-44$ & $45-54$ & $55-64$ & $65-74$ & $>=75$ \\
\hline Tuberculose respiratoire & 1.447 & 1.714 & 1.957 & 1.973 & 1,870 & 1.535 & 1.175 & .614 & .325 & .152 & .053 & .016 \\
\hline Autres tuberculoses & .492 & .460 & .143 & 109 & .097 & .088 & .077 & .049 & .034 & .021 & .010 & .001 \\
\hline Typhus & .875 & .1 .021 & .854 & .652 & .546 & .450 & .394 & .322 & .257 & .185 & .119 & .061 \\
\hline Scarlatine & 1.265 & 1.461 & .606 & .111 & .030 & .016 & .010 & .006 & .003 & .001 & .000 & .000 \\
\hline Diarrhées & .622 & .808 & .523 & .481 & .464 & .453 & .436 & .385 & .338 & .297 & .245 & .197 \\
\hline Total (1) & 5.011 & 5.809 & 4.261 & 3.447 & 3.108 & 2.625 & 2.159 & 1.419 & .987 & .674 & .437 & .281 \\
\hline Variole & .710 & .647 & .380 & .269 & .239 & .211 & .178 & .121 & .089 & .074 & .065 & .066 \\
\hline Coqueluche & .243 & .266 & .057 & .027 & .022 & .019 & .014 & .008 & .006 & .003 & .002 & .001 \\
\hline Rougeole & .068 & .123 & .041 & .012 & .004 & .002 & .001 & .000 & .000 & .000 & .000 & .000 \\
\hline Diphthérie & -.190 & -.228 & -.092 & -.011 & -.006 & -.005 & -.003 & -.002 & -.001 & .000 & .000 & .000 \\
\hline Total (2) & .833 & .809 & .386 & .298 & .260 & .228 & .190 & .129 & .094 & .077 & .067 & .068 \\
\hline Maladies cérébrales & .893 & .353 & .154 & .097 & .063 & .032 & -.003 & -.076 & -.116 & -.135 & -.106 & -.078 \\
\hline Maladies cardiaques & -.103 & -.205 & -.363 & -.408 & -.422 & -.441 & -.470 & -.528 & -.537 & -.500 & -.374 & -.230 \\
\hline Maladies pulmonaires & -.185 & .024 & .006 & -.007 & -.019 & -.023 & -.031 & -.042 & -.028 & -.046 & -.080 & .159 \\
\hline Maladies stomacales & -.505 & .056 & .309 & .326 & .326 & .324 & .318 & .273 & .224 & .176 & .094 & .022 \\
\hline Maladies rénales & -.184 & -.216 & -.248 & -.252 & -.257 & -.266 & -.273 & -.284 & -.258 & -.200 & -.124 & -.069 \\
\hline Total (3) & -.087 & .016 & .137 & -.240 & -.304 & -.368 & -.450 & -.645 & -.699 & -.685 & -.565 & -.485 \\
\hline Cancers & -.249 & -.316 & -.409 & -.432 & -.444 & -.460 & -.479 & -.518 & -.515 & -.418 & -.242 & -.110 \\
\hline Maladies infectueuses & .951 & .832 & .520 & .444 & .423 & .403 & .386 & .329 & .259 & .188 & .130 & .086 \\
\hline Maladies héréditaires & .006 & .009 & .012 & .013 & .012 & .013 & .013 & .017 & .019 & .013 & .004 & .000 \\
\hline Maladies articulaires & .096 & .115 & .128 & .111 & .088 & .070 & .060 & .048 & .036 & .024 & .013 & .005 \\
\hline Maladies de la peau & .048 & .037 & .032 & .031 & .029 & .028 & .028 & .027 & .024 & .021 & .017 & .011 \\
\hline $\begin{array}{l}\text { Morts lors de } \\
\text { l'accouchement }\end{array}$ & .048 & .059 & .069 & .069 & .059 & .047 & .037 & .032 & .015 & .006 & .003 & .001 \\
\hline Morts violentes & .149 & .232 & .197 & .141 & .101 & .070 & .045 & .009 & .000 & .004 & .013 & .029 \\
\hline Autres & .441 & .627 & -.011 & -.056 & .054 & -.036 & -.035 & .023 & .160 & .343 & .554 & .856 \\
\hline Total (4) & 1.497 & 1.598 & .522 & .302 & .196 & .117 & .035 & -.052 & -.023 & .162 & .480 & .881 \\
\hline Toutes causes de décès & 7.586 & 8.563 & 5.041 & 3.763 & 3.195 & 2.523 & 1.842 & .746 & .258 & .133 & .323 & .629 \\
\hline
\end{tabular}


La persistance opiniâtre de la tuberculose respiratoire dans les villes est bien mise en valeur par le fait que sa contribution à l'amélioration de l'espérance de vie y fut moins forte qu'à la campagne, en dépit des niveaux de départ plus élevés auxquels elle se situait ; 1,45 années contre 2,27 (voir tableau 11). Des maladies aujourd'hui plus ou moins disparues ont bien davantage contribué aux changements de la mortalité urbaine parce qu'elles partaient d'un niveau nettement plus élevé : par exemple, la scarlatine et la variole.

\section{Le déclin de la mortalité et le contraste entre villes et campagnes.}

Deux faits marquaient les villes : d'abord un niveau en général plus élevé des maladies d'insalubrité et des maladies infectieuses ; ensuite, des pourcentages plus élevés de décès masculins dus à la tuberculose respiratoire. C'est le premier groupe qui explique essentiellement le déclin de la mortalité dans la seconde moitié du siècle alors que la force du second persiste au cours du siècle. Une nouvelle étape de la présente analyse consistera donc à explorer les facteurs d'environnement expliquant ces évolutions en étudiant les villes qui furent les plus concernées. Une autre méthode pourrait cependant être mise en œuvre : nous avons suivi McKeown dans son essai pour lier ensemble les maladies et leurs causes même quand nous étions en désaccord avec plusieurs de ses interprétations. À la place, on pourrait étudier des échantillons ruraux et urbains à travers, par exemple, une analyse par facteurs, pour voir quels groupes de causes de mortalités liées à l'âge sont plus ou moins élevés selon les endroits. Nous pourrions disposer ainsi d'une démarche permettant d'identifier les facteurs divergents qui expliquent le handicap urbain.

En approfondissant l'étude des facteurs liés à l'environnement, nous aurons peut-être besoin de repenser les relations qui existent entre l'environnement et la maladie. Une démarche intéressante pourrait être un essai pour quantifier le défi créé par les maladies et pour mesurer la réponse apportée par la mortalité. Certes, l'étude des taux de mortalité permet de répondre à ces deux questions, mais il y a d'autres possibilités. D'abord, le temps : la saisonnalité de la mortalité apporte un ensemble de réponses aux risques entraînés par les diarrhées du mois d'août ; une ville qui a réussi à améliorer la sécurité quant à ce risque peut être considérée comme plus propre. De ce fait, le caractère saisonnier de la diarrhée et les variations de la mortalité en fonction de la chaleur et de la sécheresse de l'été peuvent apporter un diagnostic fort utile. Ensuite, la mortalité due aux pandémies. De même que les fluctuations météorologiques indiquent qu'il n'a pu y avoir de déclin continu de la mortalité due aux diarrhées, de même l'éclatement périodique de la variole, du choléra ou de la typhoïde introduit un élément cyclique dans l'interprétation de l'évolution de ces maladies. L'étude des villes qui, à la différence des autres, ne connaissent pas de flambées épidémiques de ces maladies, est un bon moyen de vérifier l'état de mesures sanitaires prises par les autorités telles que le quarantaine ou la vaccination.

Gerry KEARNS

Département de Géographie et Institut d'Etudes européenes de population, Université de Liverpool. 


\section{Appendice.}

\section{Note du traducteur :}

Gerry Kearns a joint à son texte anglais un long appendice, tout à fait intéressant pour les chercheurs de son pays. Il explique en effet les problèmes rencontrés pour chaque ville retenue. Pour les lecteurs d'une autre nationalité, ces notices sont beaucoup moins utiles, d'autant plus qu'il sera toujours possible de se reporter à la version anglaise ou d'écrire à l'auteur (Département de Géographie, Université Liverpool : P.O. Box 147. Liverpool L69 3BX). Il m'a semblé cependant nécessaire de publier une partie de cet appendice pour mieux faire comprendre une partie des propos de l'auteur et surtout pour bien montrer les difficultés extrêmement grandes que présente la mise sur pied d'un échantillon urbain en Angleterre. En effet, sur les 23 villes retenues, seules Leicester, Norwich, Nottingham, Preston, et, à un degré moindre Oldham, Plymouth, Portsmouth et Sunderland ne posent pas des problèmes trop aigus.

On soulignera, à partir de ces exemples, à quel point l'explosion urbaine anglaise a bouleversé les cadres administratifs du pays et créé ainsi une situation extraordinairement complexe. Par là, l'enquête menée par G. Kearns n'en a que davantage d'intérêt méthodologique.

\section{Bath.}

En 1851, dans sa définition municipale, elle a 52240 habitants; le district d'enregistrement de l'état civil en compte 69847 . En plus de la ville elle-même, on a donc 18 paroisses regroupant 15607 personnes : les notes qui accompagnent les "Tableaux de population" du recensement de 1851 expliquent que l'accroissement de leur population se justifie par leur proximité de Bath ; elles sont en train de devenir une partie de la ville telle qu'elle "fonctionne". Ces paroisses sont South Soke, Monckton Cove, Bathford, Batheaston, Swainswick et Weston; elles regroupent 8000 personnes. Il est donc clair que la ville elle-même et ses dépendances économiques directes regroupent la grande majorité de la population du district. Il n'y a aucune indication que des paroisses appartenant à des districts limitrophes aient des liens étroits avec Bath.

En 1901, la ville ("county-borough") compte 49839 personnes ; elle fait partie d'un district d'enregistrement de 77 604. Plusieurs localités du district peuvent être considérées comme une partie de la ville, telles Twerton (11 098 habitants) et Weston (5 964).

\section{Birmingham.}

En 1851, la définition municipale correspond à 232841 personnes et le district d'enregistrement à 173951 ; il s'agit de l'ancienne paroisse de Birmingham, qui est tout entière située à l'intérieur de la ville. Mais celle-ci a débordé hors de ces limites et la municipalité s'étend sur deux autres districts d'enregistrement : King's Norton et Aston.

King's Norton comprend la paroisse d'Edgbaston, forte de 9269 habitants, qui est une partie de la ville ("borough"), et le hameau de Smethwick -8379 habitantsdont la croissance démographique peut être en partie attribuée à la proximité de Birmingham. Le reste du district d'enregistrement représente 13223 habitants sur un total de 30871 pour King's Norton. La majorité du district d'enregistrement 
peut donc être considérée comme faisant partie de manière fonctionnelle de Birmingham. On doit d'autant plus retenir ce district dans son ensemble sous la définition de Birmingham, que les parties que l'on peut en identifier comme liées à la ville, sont en train de se développer beaucoup plus vite que le reste du district. En 1851, la population d'Edgbaston représente 2,3 fois celle de 1831 et celle de Smethwick a fait plus que tripler, alors que pour le reste du district l'augmentation n'est que de 1,3 fois. Il y a donc une influence croissante de Birmingham sur l'ensemble de ce district.

Le district d'enregistrement d'Aston fait plus clairement partie de Birmingham. Sa population de 66852 personnes vient des paroisses de Aston, Cudworth, Wishaw et Sutton-Coalfield. Une grande partie de la population d'Aston : 49621 sur 61281 , fait partie de la ville ("borough") de Birmingham et l'accroissement d'ensemble de la paroisse est expliqué comme dû à la proximité de Birmingham.

En 1901, le "bourg-comté" ("county borough") de la ville de Birmingham compte 522204 habitants; il comprend Edgbaston : 26 486, Harborne : 10113 et Balsall Heath : 38827 , qui font partie du district d'enregistrement de King's Norton, la paroisse de Birmingham qui est tout entière dans le district de Birmingham, et celle d'Aston qui fait partie du district d'Aston. À cette date, nous pouvons considérer que Smethwick : 54 539, qui relève du district de King's Norton, fait partie de la conurbation de Birmingham. À l'intérieur de celui d'Aston, écrivent les rapporteurs du recensement, "l'accroissement de population de la paroisse d'Erdington Civil doit être principalement attribué à sa proximité de la ville de Birmingham ; il faut donc ajouter ses 16368 habitants à ceux de la ville. Au tournant du siècle, les banlieues de Birmingham s'étendent au sud-est jusqu'à Solihull; à l'intérieur du district de ce nom, qui regroupe 48618 habitants, Yardley (qui comprend Sparkbrook) passe de 17141 à 33946 habitants de 1891 à 1901, et cet accroissement est attribué à la proximité de Birmingham".

\section{Bolton.}

En 1851, la ville de Bolton comptait 61171 habitants mais le district d'enregistrement en incluait 114712 . On n'a dans les notes qui accompagnent le recensement aucune information précise sur les caractéristiques de cette zone. La ville comprend les communautés municipales de Great Bolton, Little Bolton et Haulgh qui font entièrement partie du district d'enregistrement. La majorité du reste de la population réside dans de denses peuplements industriels qui se trouvent à quelques "miles" de Bolton.

En 1901, la population de la ville, qui atteint 168215 habitants, représente la majorité du district d'enregistrement où l'on en dénombre 257587 ; en outre la paroisse de Farnworth : 25925 habitants fait clairement partie de la zone bâtie de la ville.

\section{Bradford.}

En 1851, avec 103778 habitants la ville n'est qu'une partie du district : 181964 . C'est un autre exemple de ville qui pose de grands problèmes. En effet, les notes qui accompagnent le tableau du recensement, ne donnent que de brèves indications topographiques sur la population des différentes localités. La ville contient les communautés municipales de Bowling, Bradford, Great Norton, Little Horton et Manningham, qui se situent toutes à l'intérieur du district d'enregistrement. 
En 1901, la "ville-comté" de Bradford s'étend sur deux districts : Bradford 228625 habitants- et North Bierley -129 067-. C'est dans le West Riding que les relations entre les districts d'enregistrement, les paroisses et les unités administratives sont probablement les plus compliquées ; en outre, il y eut de nombreux changements entre les différents recensements : c'est ainsi que deux districts d'enregistrement échangèrent de nombreuses paroisses entre 1891 et 1901 . Au début de 1892, Bradford céda 16 paroisses, groupant 146505 habitants en 1901, à North Bierley; ce dernier district céda à Bradford le 1er octobre 18984 zones géographiques où vivaient 14446 personnes ; North Bierley céda également au district d'Halifax une partie de la paroisse de Clayton -2603 habitants- le 1er juin 1895, et une partie de Wike -389 habitants- le 1er février 1897. L'enregistrement de Bradford faisait entièrement partie de la "ville-comté"; le reste de sa population 51143 habitants-faisait partie de ce qui avait été transféré à North Bierley. En ce qui concerne le reste du district de North Bierley, plusieurs des paroisses qui le constituaient se trouvaient à une certaine distance des zones bâties : telles Drighlington -11 530 habitants-, Cleckheaton -12 524-, et Hunsworth -1 346-, qui se situaient entre 3 et 4 miles au sud-est de la ville. Néanmoins, la paroisse de Shipley, dont la population était de 25573 habitants, principalement dans Saltaire, doit être probablement considérée comme faisant partie de la ville à cette date et ce serait sans doute une erreur de l'exclure de notre définition de Bradford, tout comme les localités de banlieue cédées au district de North Bierley.

\section{Brighton.}

En 1851, Brighton n'est pas dotée d'une charte lui accordant une municipalité ; mais elle est une ville "parlementaire". Celle-ci compte 69673 habitants et son district d'enregistrement 65 569. Ce district comprend la ville "administrative" de Brighton, connue également sous le nom de paroisse de Brighthelmstone. Le reste de la ville "parlementaire" comprend la paroisse de Hove : 4104 habitants, qui est une petite partie du district d'enregistrement de Steyning, fort de 16687 habitants.

En 1901, la ville-comté de Brighton comprend la paroisse civile de Brighton qui correspond au district d'enregistrement du même nom ; soit 102320 habitants, et la paroisse de Preston : 21158 habitants qui fait partie du district de Steyning : 80789 habitants. La ville de Hove doit sans doute être également considérée comme une partie de l'aire bâtie continue de Brighton ; elle représente 29695 habitants du district de Steyning. C'est une bonne raison pour considérer que les deux districts font partie de la ville de Brighton en 1901.

\section{Bristol.}

La ville de Bristol compte en 1851137328 habitants; elle s'étend sur deux districts d'enregistrement : Bristol, avec 65716 personnes, et Clifton : 77950 ; le premier correspond à la vieille ville de Bristol ; le deuxième comprend Clifton 7634 habitants- qui est une partie de la ville de Bristol, Saints-Philippe et Jacob 24 961-qui est une paroisse extérieure de Bristol - Saint-Jacques et Saint-Paul qui sont les dépendances extérieures de paroisses de la ville mais qui sont incorporées dans ses quartiers ("wards"), 7610 de leurs 7935 habitants se trouvant à l'intérieur des frontières de la ville ; la paroisse de Horfield : 998 personnes, plus 223 soldats dans des baraquements, qui était à l'origine une extension de Bristol, mais qui n'a jamais fait formellement partie de la ville; et Stoke Bishop Tything, 4213 habitants dont "la plus grande partie $(3809)$ vivent à l'intérieur des limites de Bristol". Le reste de la ville, soit 17598 habitants, fait partie du district d'enregis- 
trement de Bedminster, qui appartient au comté de Somerset et représente presque la moitié de la population de ce district : 38143 habitants, et l'essentiel de la paroisse de Bedminster : 19434 habitants. Rien n'indique que le reste du district de Bedminster doive être considéré comme une partie de la ville de Bristol ; celuici n'a donc pas été inclus dans notre échantillon urbain, en vertu des règles qui avaient été énoncées, puisqu'il représente moins de $50 \%$ du total.

En 1901, la "ville-comté" de Bristol a 328945 habitants. Le district d'enregistrement donne une bien meilleure définition de la ville. Mais l'essentiel des changements sont survenus en fin de période et nous avons donc été obligé d'introduire les naissances et les décès des districts d'enregistrement de Bedminster et de Barton Regis dans ceux de Bristol, le district d'enregistrement de Clifton ayant pris le nom de Barton Regis le 1er avril 1877. Le district d'enregistrement de Bedminster de 1891, qui compte alors 77574 habitants, perd donc, par suite de cette modification, la paroisse de Bedminster : 45627 habitants, ainsi que des parties d'Easton, de Long Aston, de Portburry et de Bedminster -Without, soit 7678 habitants. En 1899, la séparation de cette zone qui groupait en 1891 53406 habitants, laisse un district rural croupion, désormais appelé Long Aston, où l'on trouve 24138 habitants en 1901. Par ailleurs, Barton Regis perd des zones, dont la paroisse de Clifton, qui représentent 177995 sur les 193094 qu'il comptait en 1891 .

\section{Leicester.}

En 1851, la population de la ville compte 60584 habitants qui vivent entièrement à l'intérieur du district d'enregistrement.

En 1901, le district d'enregistrement, la paroisse et le comté ont les mêmes limites ; leur population est de 211579 habitants.

\section{Merthyr Tydfil.}

En 1851, elle n'a pas encore de charte municipale mais 63080 habitants vivent à l'intérieur de la ville "parlementaire", qui se trouve presque entièrement dans le district d'enregistrement ; celui-ci inclut la paroisse de Merthyr Tydfil 34977 habitants-, et d'autres paroisses qui dépendent de mines ou d'usines métallurgiques. La ville parlementaire comprend la paroisse d'Aberdare : 14999 habitants, une partie de celle de Merthyr Tydfil, réunie à Forest, Taff et Cynon -soit au total 45844 habitants-et le hameau de Coed-y-Cymmar : 2237 habitants, qui appartient à la paroisse de Vainor.

En 1901, le district urbain ("Merthyr Urban District") a 69228 habitants, ce qui est un peu plus de la moitié de la population du district d'enregistrement : 135540 , lequel comprend la ville complètement indépendante d'Aberdare : 53365 habitants.

\section{RÉSUMÉ}

L'article est une recherche sur les causes de la mortalité en Angleterre et au pays de Galles dans la deuxième moitié du XIXe siècle, sur leur évolution et sur les différences trés marquée entre villes et campagnes : le handicap urbain. Le problème est de bien mesurer les causes de la mortalité, ce qui suppose une recherche plus approfondie que celles qui ont été menées jusqu'ici sur le rôle des différentes maladies et 
sur la définition des circonscriptions administratives. L'article a donc une caractéristique méthodologique trés marquée. Pour mener la recherche, il a fallu en effet construire un échantillon urbain - ce qui est fort difficile, comme le montre l'appendice - et un échantillon rural. La recherche est à continuer : il reste que la différence essientielle pour les adultes entre les villes et les campagnes s'explique par la tuberculose respiratoire. Quant à l'écart de l'espérance de vie, il reste marqué, les campagnes ayant également connu une amélioration de leur mortalité.

\section{SUMMARY}

This paper is a research on the causes of the mortality in England and Wales during the second half of nineteenth century, their evolution and the very large difference between the cities and the country, what is the urban penalty. The difficulty is the measure of the causes of mortality; we must achieve a most indepth research on the influence of each disease and on the definition of the administrative districts. Therefore, this paper is caracterized by a great methodological effort. To make the research, it was necessary to construct an urban sample -and it is a particularly difficult work, as it is showed in the appendix-, and a rural sample. The research must be continued, but to-day it is clear that the great difference for the adults between cities and countries must be explained by respiratory tuberculosis. As for the gap in the life expectancy, it is always large, because the country has known also an improvement of his mortality. 